\title{
Tip Position
}

\author{
Steve Hill and Nancy L. Moureau
}

\section{Abstract}

This chapter provides insight into catheter tip location and review of vascular access devices from PIV to central venous non-tunnelled and tunnelled catheters, implanted ports and dialysis catheters. An overview of the guidelines related to tip location is discussed. The dynamic relationship between catheter tip location and how this influences complications during the procedure and post-insertion is examined. The various methods of confirming CVAD tip location are presented, including ECG (i.e. echocardiography) versus traditional means of X-ray, exploring patient implications of intraprocedural real-time tip location, reduced radiation dosage and incidence of manipulations. Case studies illustrating some of the challenges clinicians face when placing CVADs are incorporated into the discussion.

S. Hill

The Christie NHS Foundation Trust, Manchester, UK

Precision Vascular and Surgical Services Ltd,

Manchester, UK

e-mail: info@precisionvascular.co.uk; steve.hill@christie.nhs.uk

N. L. Moureau $(\bowtie)$

PICC Excellence, Inc., Hartwell, GA, USA

Menzies Health Institute, Alliance for Vascular Access Teaching and Research (AVATAR) Group, Griffith University, Brisbane, QLD, Australia e-mail: nancy@piccexcellence.com

\section{Keywords}

Superior vena cava Cavoatrial junction Tip location · Thrombosis · Arrhythmias Electrocardiogram $\cdot$ Terminal catheter tip Malposition · Complications · Central venous catheter

\subsection{Introduction}

A great deal of research, time and energy has gone into the subject of catheter tip position, yet it is still yielding debate and controversy. Many of the guidelines are united in placement of optimal tip position of a central venous access device. The associations are the Infusion Nursing Society (INS), Association of Anaesthetists of Great Britain and Ireland (AAGBI), European Society of Parenteral and Enteral Nutrition (ESPEN) and British Society for Haematology (BCSH) (Table 7.1).

The reason for the focus on tip position is that malposition of the catheter tip is associated with an increased risk of complications. These may occur at the time of insertion (atrial fibrillation, ventricular tachycardia or other arrhythmias, atrial wall damage, catheter malfunction) or postinsertion (catheter malfunction, vessel erosion, venous thrombosis). Misplacement of catheter does not only mean the incorrect vein but also includes catheters inserted in extra-venous positions such as in the arterial system, mediastinum, 
Table 7.1 Guidelines for terminal tip placement (Gorski et al. 2016a; Pittiruti et al. 2009)

\begin{tabular}{l|l}
\hline INS (2016) & $\begin{array}{l}\text { Lower third of the superior vena cava or } \\
\text { cavoatrial junction }\end{array}$ \\
\hline $\begin{array}{l}\text { AAGBI } \\
(2016)\end{array}$ & Lower SVC upper right atrium \\
\hline $\begin{array}{l}\text { ESPEN } \\
(2006)\end{array}$ & $\begin{array}{l}\text { Lower third of the superior vena cava or } \\
\text { at the atrio-caval junction }\end{array}$ \\
\hline $\begin{array}{l}\text { BCSH } \\
(2006)\end{array}$ & $\begin{array}{l}\text { Distal superior vena cava or upper right } \\
\text { atrium }\end{array}$ \\
\hline
\end{tabular}

pleura, trachea, oesophagus, etc. (Gibson and Bodenham 2013). This chapter will focus on tip placement, controversies, guidelines and other considerations when selecting the tip position for centrally placed intravenous devices.

\subsection{Vascular Access Device Terminal Tip Positioning}

Correct terminal tip positioning of a vascular access device is determined by manufacturer specifications as well as regulatory and guideline evidence-based criteria. Indications for each VAD also contribute to the choice of device, function and tip position based on treatment plan.

- Peripheral catheters remain in the periphery with terminal tip below the level of the axillary vein for upper extremity placement. The terminal tip of a peripheral catheter does not enter the chest or torso and, therefore, does not require any verification following insertion (Gorski et al. 2016a).

- Central venous access devices (CVADs), as their name implies, are inserted with the terminal tip entering central circulation and advancing towards the heart. Except for haemodialysis catheters, terminal tip placement of all CVADs is in the vena cava. For upper extremity insertions, the terminal tip is advanced to the superior vena cava; for lower extremity insertions, the terminal tip is advanced to the inferior vena cava (Campisi et al. 2007; National Association of Vascular Access Networks 1998; Scott 1995; Vesely 2003).

\subsection{Anatomy}

A CVAD by definition has the distal tip of the catheter within the central veins. INS suggests that ideally a central venous catheter should have its tip situated in the lower third of the superior vena cava at or near the cavoatrial junction (Gorski et al. 2016a). Firstly, let's review venous anatomy relevant to tip placement; to consider tip position and recognise misplacement, we must be clear in our knowledge of the venous system. Venous structures, such as vein walls (tunica intima, tunica media and tunica adventitia), are thinner and more fragile than arteries, making them more prone to iatrogenic injury, and their longitudinal organisation of vein layers means that tears in the vein walls tend to extend longitudinally (Gibson and Bodenham 2013).

The larger venous vessels within the thorax relevant to vascular access include axillary, subclavian, brachiocephalic, internal jugular, superior vena cava, iliac, femoral and inferior vena cava (Fig. 7.1). In the adult, the superior vena cava's average length is $7 \mathrm{~cm}$ but varies from 5 to $11 \mathrm{~cm}$ (Verhey et al. 2008). The brachiocephalic veins, from the right side, is positioned at a steeper angle and is around $2.5 \mathrm{~cm}$ in length, and the left brachiocephalic around $6 \mathrm{~cm}$ in length joins the right brachiocephalic and the SVC at a less acute angle than the right brachiocephalic. CVAD insertions from the left brachiocephalic that are not long enough to drop down into the SVC can abut the wall of the SVC increasing the risk of thrombosis and erosion (Gibson and Bodenham 2013).

Other relevant vasculature also includes the azygos system which drains venous blood from the upper thorax and abdominal wall terminating in the SVC (Tortora and Derrickson 2006). The azygos vein drains some of the lumbar and posterior intercostal region and also connects to the posterior aspect of the IVC, level with the first or second lumbar vertebrae, to the IVC (Gibson and Bodenham 2013). Catheter tips placed in the azygos vein viewed on standard anterior-posterior X-ray can appear short at first glance, but with closer inspection and by performing a lat- 
Fig. 7.1 Anatomy of the arm and chest (used with permission of N. Moureau, PICC Excellence)

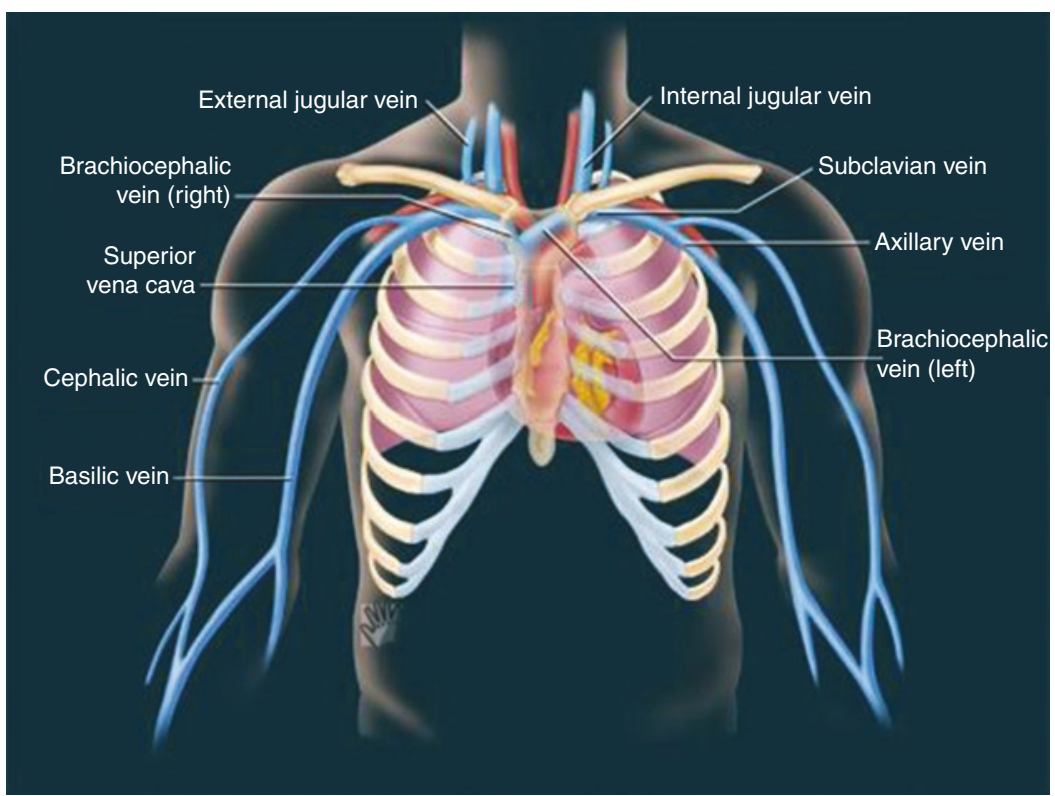

eral X-ray film, the catheter can be distinguished as it changes course from the vertical SVC and may turn abruptly moving posteriorly and inferiorly. Smaller vessels such as the superior intercostal veins are important as a catheter may migrate into these during the insertion of upper thoracic CVADs. VADs inserted via the femoral veins should have their tips placed in the inferior vena cava (IVC) above the level of the diaphragm (Gorski et al. 2016a). During femoral insertion, the catheter may migrate into smaller veins during insertion including lumbar, iliolumbar renal, suprarenal, common iliac, hepatic and inferior phrenic veins (Gorski et al. 2016a; Tortora and Derrickson 2006). The IVC drains blood from the lower part of the body, ascends along the anterior vertebral column and is around $2.5 \mathrm{~cm}$ wide (Gibson and Bodenham 2013).

Arm anatomy-Moving away from the larger more central veins, there are the axillary, brachial, cephalic and basilic veins of the upper arm. Moving distally, the veins start to become smaller in diameter, and blood flow is reduced. When considering veins of the upper arm for PICC and midline placement, the main vessels include basilic, brachial and cephalic veins. Moving distally to around the antecubital area, the median cubital and cephalic are often prominent veins; smaller vessels include the accessory cephalic, brachial and intermediate antebrachial. Within the forearm, there are the branches of the basilic, cephalic and median veins together with radial, posterior/anterior ulna and accessory cephalic veins. The dorsum of the hand includes metacarpal veins and the dorsal venous arch (DVA) which has many tributaries; the medial aspect of the DVA leads the flow to the cephalic veins, and the lateral aspect flows to the basilic veins (Fig. 7.2). Anatomy is not universal, and variations exist from person to person. Assessment is the key to identifying anatomical variations and optimal vein and VAD site. Ultrasound is an excellent visualization and enhances assessment of veins, arteries and nerves.

Cannulation-Insertion of a short PIV has been described as the most frequent invasive procedure in acute care, with the most common complications being dislodgement and phlebitis (2.3-67\%) but has a particularly low incidence of infection relative to other vascular devices (0.5/1000 catheter days) (Bertoglio et al. 2017). Catheter sizes from $20 \mathrm{G}$ to $22 \mathrm{G}$ are recommended for adult patients if possible (Gorski et al. 2016a). The forearm is strongly recommended (Bertoglio 


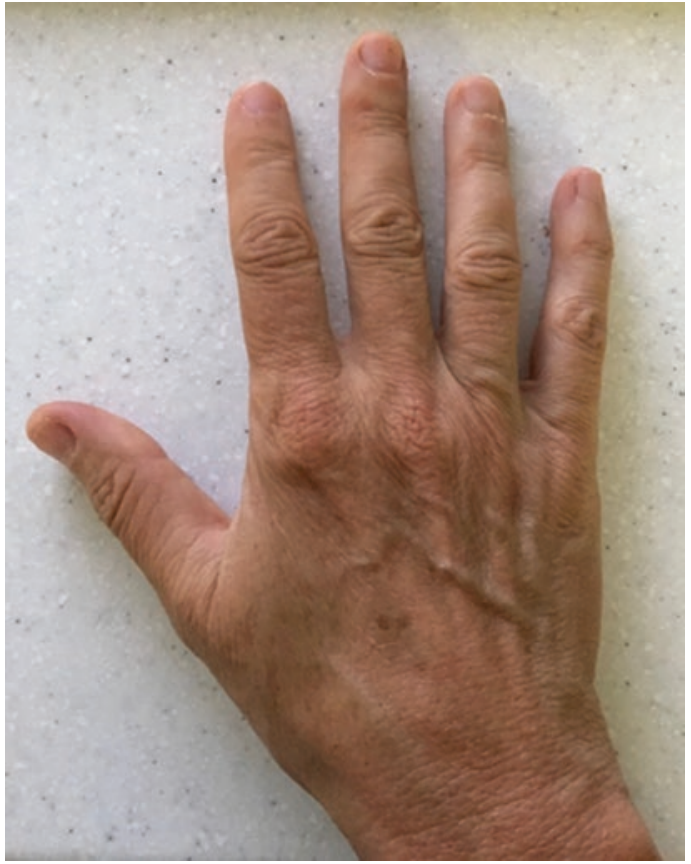

Fig. 7.2 Hand and dorsal venous arch (used with permission of N. Moureau, PICC Excellence)

et al. 2017). Selecting the optimal site for placement of PIV impacts upon the functional lifespan of the device, Wallis et al. (2014) identified that the forearm is the optimal position for the longest dwell time; this area is also associated with decreased pain (Gorski et al. 2016a). Risk factors for patients receiving chemotherapy include placement of the catheter in the hand and antecubital fossa and upper arm when compared to the forearm (Gorski et al. 2016a). A thorough assessment is necessary, not only of the suitability of the vessels but to the length of the PIV related to the depth of the vein. INS recommends two-thirds of the catheter be placed in the vein to establish safety (Gorski et al. 2016a). Consider the use of longer PIV lengths or midlines if a safe amount of catheter cannot be established within the vessel. An ultrasound-guided cannula placed in the upper arm is at increased risk of extravasation and infiltration (Gorski et al. 2016a). PIV sites such as the wrist and antecubital should be avoided, as areas of flexion they have increased rates of complications (Gorski et al. 2016a). Furthermore, patients planned for future mastec- tomy surgery or dialysis fistula should not have PIVs placed over joints, forearm is preferred (Bertoglio et al. 2017). Due to the relatively short length of most PIVs, site selection becomes an important determinant of tip position, as the insertion site and tip are within a short distance of each other.

Lower limbs should be avoided for insertion of intravenous devices unless absolutely necessary as they are at increased risk of tissue damage (Gorski et al. 2016a), alternative sites should be explored preferably. PIV position should ideally be in the non-dominant arm, and ventral surfaces of the wrist may increase pain and risk of nerve damage and thrombosis (Gorski et al. 2016a; RCN 2016). For many years, first attempt PIVs have been made on the dorsal aspect of the hand, but with advent of infrared and ultrasound technology, non-visible or difficult to palpate veins are now easier to assess and access. Veins of the forearm can lie slightly deeper than veins on the dorsum of the hand and overlying the antecubital area and enhanced by aids such as visualisation technology. In situations where intravenous access is needed, more urgently peripheral catheters may be inserted into the external jugular (Gorski et al. 2016a) or leg veins by a suitably trained clinician and when treatment is less than 4 days (Chopra et al. 2015).

Midline catheters are used for appropriate peripheral solutions; the Michigan appropriateness guide for intravenous catheters (Moureau and Chopra 2016) suggests that they are preferred for solutions up to 14 days but may be used in a manner consistent with the clinically indicated removal of peripheral catheters. Midline catheters come in a variety of length and diameters; INS principles should be followed when selecting a VAD with the smallest outer diameter (Gorski et al. 2016a). The midline is placed into the basilic, cephalic or brachial veins, by way of sterile procedure similar to PICC placement, with the exception of need to verify terminal tip since the midline remains in the peripheral veins (Gorski et al. 2016a). Adults and older children should have the tip of the midline placed at the level of the axilla and distal to the shoulder (Gorski et al. 2016a). 
The axillary vein by definition is:

the continuation of the basilic and brachial veins running from the lower border of the teres major muscle to the outer border of the first rib where it becomes the subclavian vein. (Farlex Medical Dictionary 2012)

The teres major muscle is attached to the scapula and to the humerus and is the most inferior positioned muscle of the muscles connected to the scapula; its function is to aid adduction of the arm.

One of the advantages of the midline compared to CVADs is that chest X-ray or ECG confirmation is not required. When the midline is appropriate, it may be more time efficient for the vascular access clinician and a more costeffective solution, as midlines are often a comparably cheaper option versus other CVADs. Like all devices, it must be inserted according to the manufacturer's instructions; if there are concerns during insertion, impaired blood return or resistance to flushing an X-ray or replacement device should be considered (Alexandrou et al. 2011). As the tip position of a midline is placed at the level of the axilla where extravasation could be a risk, thus placing other anatomical structures at risk such as arteries and nerves, if fluid escapes into the tissues, it may be harder to detect than in the forearm or hand (Hadaway 2000).

Central venous catheter tip locationCVAD tip position for many years has been confirmed by the means of a radiograph or chest X-ray (CXR). In recent years, electrocardiograph (ECG)-based systems have been developed to confirm catheter tip position, some of which also have additional navigational elements. In this section we will discuss traditional radiographic techniques, the evolution of current practice and the adoption of tip confirmation technology.

Undertaking CVAD insertions under direct $\mathrm{X}$-ray screening/fluoroscopy offers real-time visualisation of elements of the insertion, such as position of the guidewire tip deep in the thorax, migration and immediate confirmation of tip position. This has been, and is still for many institutions, the mainstay for CVAD insertions because of the advantages offered and because in many hospitals CVAD insertions still fall within the remit of interventional radiologists. Undertaking a postprocedure chest X-ray following a fluoroscopic insertion should be decided on an institutional basis; this is mainly for exclusion of pneumothorax as assessment is limited with fluoroscopy only; ultrasound sliding lung and assessment of pneumothorax may also be considered. A similar technique, but not radiographically robust as interventional suites, is for a CVAD insertion to take place in theatre or procedural area with assistance of a portable X-ray system or C-arm image intensifier. The technology has advanced, and image quality has improved; however portable systems remain inferior to fluoroscopy imaging. Both the fluoroscopy and C-arm approach are relatively expensive models of practice due to the capital and maintenance costs related to the systems.

Other models of insertion practice involve insertion in a designated area, or a multipurpose room that is suitable also for CVAD insertion. Using this, tip location can be confirmed during insertion using ECG confirmation system or by sending the patient to X-ray. The latter X-ray approach is prone to increased risk malposition as confirmation of tip position is sought following insertion. Lastly, many services place PICCs by the bedside, but this approach too is prone to higher insertion-related malposition unless using tip confirmation technology versus postprocedure X-ray confirmation. As we continue to see the adoption of ECG guidance systems, fluoroscopy remains inextricably linked to vascular access services for patients with complex congenital or disease-related anatomy, stenosis, etc., where more advanced insertion techniques are required. However, cost associated with fluoroscopy and the need to schedule and transport patients makes bedside PICC with ECG guidance more attractive and reasonable given the level of precision and accuracy associated with ECG.

\subsection{ECG Tech Development}

The concept of confirming catheter tip position by alternative means to X-ray is not a new one. As the knowledge and skills of identifying cardiac electrical impulses have evolved over the 
last century, this has given us the fundamental principles of ECG that we use in clinical practice today. Using this knowledge and transferring it into clinical applications allow us in today's clinical practice to confirm tip position of a CVAD using ECG technology.

Carlo Matteucci a professor of physics in Florence initially identified electrical impulses relating to cardiac pulsation (Matteucci 1842). Marey (1876) used an electrometer to record cardiac electrical activity from an exposed frog heart. Soon after Sanderson and Fredrick identified the two phases of cardiac electrical activity (BurdonSanderson and Page 1878) and at the turn of the century, Arthur Cushny at University College London reported the first case of auricular fibrillation (atrial fibrillation), making the connection between an irregular pulse and atrial fibrillation (Cushny and Edmonds 1906). Augustus Waller published the first human electrocardiogram, using a capillary electrometer, where the surface electrodes were strapped to the front of the chest and back and used saline jars where the limbs were immersed in saline solution. He identified that 'each beat of the heart is seen to be accompanied by an electrical variation' (Bestman and Creese 1979; Waller 1887).

Much of Waller's work was overshadowed by his colleague Willem Einthoven (1895) who developed the electrical waveforms we know today as P-, Q-, R-, S- and T-waves and eventually received a Nobel Prize for his work in the discovery of the mechanism of the electrocardiogram. The prize was awarded in 1924; sadly Waller could not be considered for the prize as he died in 1922.

Hellerstein continued the practical application of electrocardiography and in 1949 monitored the heart's electrical action at the distal catheter tip using saline within the catheter lumen, as an agent to conduct the cardiac electrical signals, using an adapter on the proximal end of catheter and an ECG monitor. He noted a pronounced increase in the P-wave as the catheter tip entered the right atrium (Hellerstein et al. 1949). Several years have elapsed since these early experimental technologies gave birth to commercially dedicated systems.
Today there are a number of systems commercially available that confirm catheter tip position, and there is an increasing trend to include or have optional navigational functionality.

The catheter tip confirmation systems available today are fundamentally based around the principles of ECG developed by the pioneers and predecessors such as Augustus Waller, who paved the way for our practice today. Focusing upon the cardiac impulses, the current systems are reliant upon the sinoatrial (SA) node and atrial electrical impulses. As the SA node fires, it triggers depolarisation of the atria and contraction of the two chambers, which on an ECG is represented by the P-wave of the normal P-, Q-, R-, S- and T-wave ECG complexes (Fig. 7.3).

In a sinus rhythm, where conduction is following the normal electrical pathway through the heart, the P-wave can be easily distinguished. Certain heart rhythms affect the atrial conduction pathway and so affect the transmitted P-wave on the ECG. Atrial flutter and atrial fibrillation are conditions where the atrial electrical conduction is disrupted; this is represented on the ECG in $\mathrm{P}$-wave distortion. When the $\mathrm{P}$-wave is not discernible, then other means of catheter tip confirmation is required such as a CXR. It is important that in patients with extreme tachycardia or with a pace-

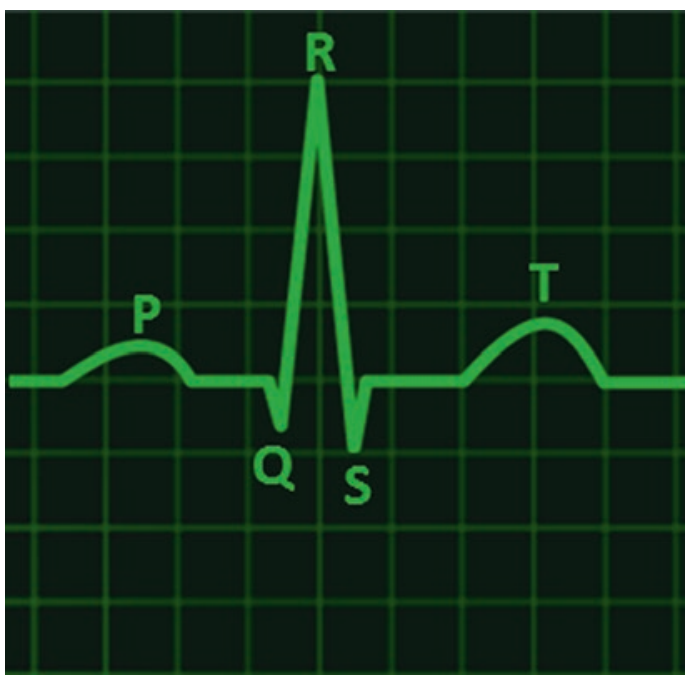

Fig. 7.3 Electrocardiogram normal sinus rhythm (used with permission of N. Moureau, PICC Excellence) 
maker in situ, where the P-wave may not be discernible, an alternative method of tip confirmation is used. The instructions for use on most systems will require X-ray confirmation in these clinical instances. In some of these situations, changes in P-waves can be identified, aiding the clinician to achieve satisfactory tip position. However, X-ray confirmation is still required according to many systems' instructions. Technological advancements will soon develop systems that can provide tip confirmation, even when the clinician cannot readily identify the $\mathrm{P}$-wave, such as when the heart is in atrial fibrillation.

The consoles are connected to the catheter via a sterile component that facilitates impulse conduction. ECG-alone systems detect the electrical impulses at the distal tip of the catheter during placement; the impulses are then processed by the console and presented in a graphical display for the operator to interpret. On the consular display screen, there are usually two ECG waveforms, one will be the standard ECG detecting cardiac electrical activity from the skin surface, and the other is an ECG detecting cardiac impulses from the internal catheter at the distal tip, the intracavity ECG.

The electrical impulses can be detected via the saline column in the lumen of the catheter or via a stylet/wire; both ways conduct the impulses from the distal tip of the catheter. The stylet system usually requires a crocodile clip, or an alternate means, to enable conduction from the stylet to the console or to a monitoring unit.

During a successful tip placement, the changes in ECG signal will firstly show a slight elevation in the P-wave as the catheter enters the SVC and moves towards the heart.

The P-wave continues to increase in size as it progresses towards the atria, which reaches its maximum amplitude at the cavoatrial junction.

As the catheter crosses the threshold of the cavoatrial junction, a portion of the atria and the SA node is now slightly superior to the tip of the catheter. This results in a reduction in the $\mathrm{P}$-wave height and a portion of the $\mathrm{P}$-wave becoming negative; a biphasic P-wave can be observed.

If the catheter is advanced, a further reduction of the $\mathrm{P}$-wave height is observed together with an increasing biphasic signal.
The action of placing a catheter tip using ECG catheter tip confirmation system is a process, not just a matter of obtaining a particular waveform and leaving the catheter tip at that point. The process must include certain steps and acknowledgements of different waveforms to ensure that the catheter is indeed in the correct position. Once the catheter tip starts its journey down the SVC, the P-wave of the waveform starts to rise; at this time the catheter should be advanced slowly, allowing time for the system to translate the information received at the distal tip of the catheter into a graphical representation on the screen for the clinician to interpret. Cautious advancement of the catheter will see the P-wave begin to rise as the tip moves down the SVC to the cavoatrial junction.

Initial systems only offered ECG to provide tip confirmation, but evolution of this technology has seen the development of tracking and navigational options to provide added insight to the inserter of the position and direction the catheter during insertion and not just tip confirmation. Tip tracking technology may use magnetic or electromagnetic methods to track the movement of the catheter tip. Not all systems can be used with all different catheter types; some manufacturers limit the use of their systems to be used solely with their own products. PICCs have a longer venous pathway to arrive to their destination and have a greater opportunity to become misplaced compared to a non-tunnelled CVC being placed into the RIJ, where the pathway to the SVC and CAJ is a much straighter course and allows the passage of the catheter without guidance easier (AAGIB 2016). ECG only may be more suited for routine RIJ central venous insertions and navigation for other CVAD insertions.

Some tip tracking systems work by placing a T-piece sensor upon the chest of the patient, and then as the PICC is passing from the arm through the axilla towards the central veins, the tip can be tracked and displayed on the screen.

One system which uses alternative technology approach is VPS G4 ${ }^{\mathrm{TM}}$, which uses Doppler signal to support the navigation. The Doppler signal is derived from transmitting bursts of fixed high-frequency energy from the stylet (illustrated 
by the grey wave fronts above) and then listening for the reflected sound from the blood particles. Blood particles flowing towards the stylet will generally reflect a slightly higher frequency (illustrated by the blue reflected wavelets above Fig. 7.4), whilst particles moving away from the stylet will reflect a slightly lower frequency back to the stylet sensor (shown by the magenta reflected waves (Fig. 7.5)).

The system can detect if the catheter is progressing with the flow of the venous blood, or if the catheter has migrated into the wrong vein, i.e. internal jugular, contralateral brachiocephalic or subclavian, by sensing retrograde blood flow and by displaying this on the console for the inserter see.

ECG-Walker et al. (2015) undertook a systematic review of RCTs (729 patients), looking at ECG only versus landmark/anatomical insertions with post-procedure CXR (Walker et al. 2015). It was identified that slightly higher complications

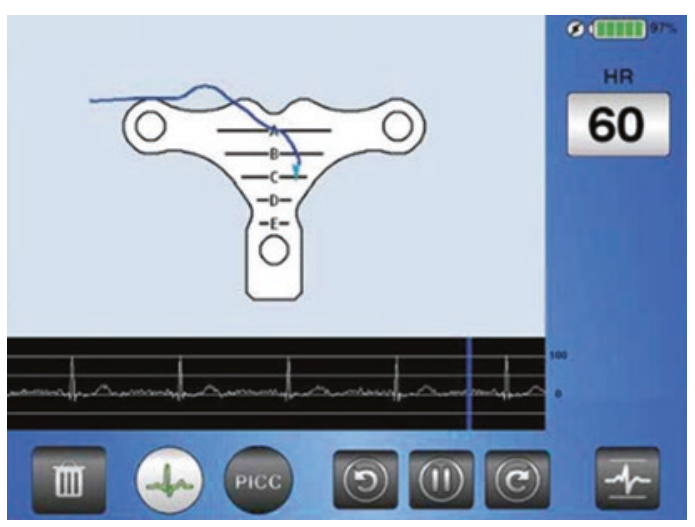

Fig. 7.4 Tip tracking and navigation technology (used with permission of Teleflex) occurred in the ECG arm in two of the studies reviewed; however these were mainly arterial punctures or haematoma. The authors point out that these complications pertain more towards insertion technique and use of ultrasound rather than related to the use of ECG. The review identified that ECG-based method was eighth times more effective than anatomy guided. The authors concluded that ECG confirmation appears a suitable replacement for post-procedural CXR confirmation (Walker et al. 2015).

Yan Jin Liu (2015) studied two groups of patients using conventional PICC placement versus ECG-guided PICC placement, followed up then with CXR (Yan et al. 2017). In this randomised controlled study of 1007 patients, first attempt success was $89.2 \%$ in the ECG group versus $77.4 \%$ in the anatomical landmark group. The study also showed increased accuracy with ECG with right-sided placements versus left-arm approach.

The UK Royal College of Nursing Standards advises that tip position can be confirmed radiographically or with ECG guidance (RCN 2016). INS (2016) suggests post-procedure radiograph imaging is not necessary if alternative tip location technology confirms tip placement (Gorski et al. 2016a).

Ultrasound-In recent years there has been increasing interest in the role of ultrasound during tip placement; clinicians have examined its efficacy of confirmation of CVAD tip position. Duran-Gehing and colleagues (2015) describe the method of identifying CVC tip position using transthoracic ultrasound, a saline flush injected via the distal lumen of the CVC whilst visualising the right atrium with the ultrasound.

Fig. 7.5 Sound waves projecting image (used with permission of S. Hill, Precision Vascular) 
If microbubbles/turbulence of the saline can be observed within the RA in $2 \mathrm{~s}$ of the flush confirms satisfactory tip position, the authors suggest this method has $96 \%$ sensitivity. Marano et al. (2014) used transthoracic ultrasound to confirm PICC tip position in 53 patients; ultrasound was undertaken by a non-cardiologist and controlled by chest radiography. Catheter tip position was identified using this method successfully in 94.3\% of cases, and 3 cases were malpositioned into the Mid-SVC; the authors suggested larger cohorts of patient to increase the validity of this method and noted that a limitation to this method is body habitus.

Weekes et al. (2016) prospectively inserted non-tunnelled catheters placed in emergency settings comparing ultrasound versus CXR for 151 patients. Four suboptimal catheter positions were detected by CXR; ultrasound identified three of these; again this study showed a shorter time to when the catheter could be used in the ultrasound arm. The authors concluded no significant difference in the accuracy between ultrasound and CXR for confirming tip position. Raman et al. (2017) compared non-tunnelled CVC tip position using transthoracic ultrasound versus CXR, monitoring the length of time before the tip position was confirmed and the catheter could be used, and identified that ultrasound confirmation allowed the catheter to be used in half of the time it took to gain CXR confirmation. This is a promising area of development regarding tip position ultrasound technology advances, as bedside ultrasound is becoming increasingly more capable and is no longer exclusively the realm of radiologists. Though limited by body habitus and patients with increased BMI, high accuracy of this approach provides another option for the experienced user, but further research and data is needed. INS (2016) reaffirms this point and suggests caution with ultrasound for CVAD tip location as its use to confirm tip location in all ages due to small sample sizes (Fig. 7.6).

However, the guidewire would not advance lower down the vessel (Fig. 7.7). The needle and guidewire were then removed. The lower vessel was then cannulated, and the guidewire was passed routinely without resistance; a fluo- roscopic image then showed the guidewire has passed into the ipsilateral subclavian vein.

After removing the needle over the wire, the wire was then manipulated into satisfactory position (Fig. 7.8).

The guidewire continued into a satisfactory position, and the procedure continued routinely until the catheter was placed, and the catheter

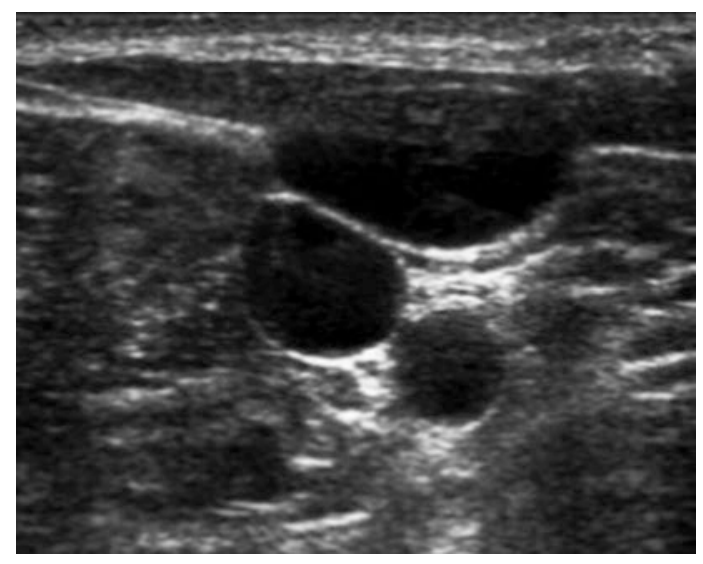

Fig. 7.6 Ultrasound image of duplicate/fenestrated internal jugular veins (used with permission of S. Hill, Precision Vascular)

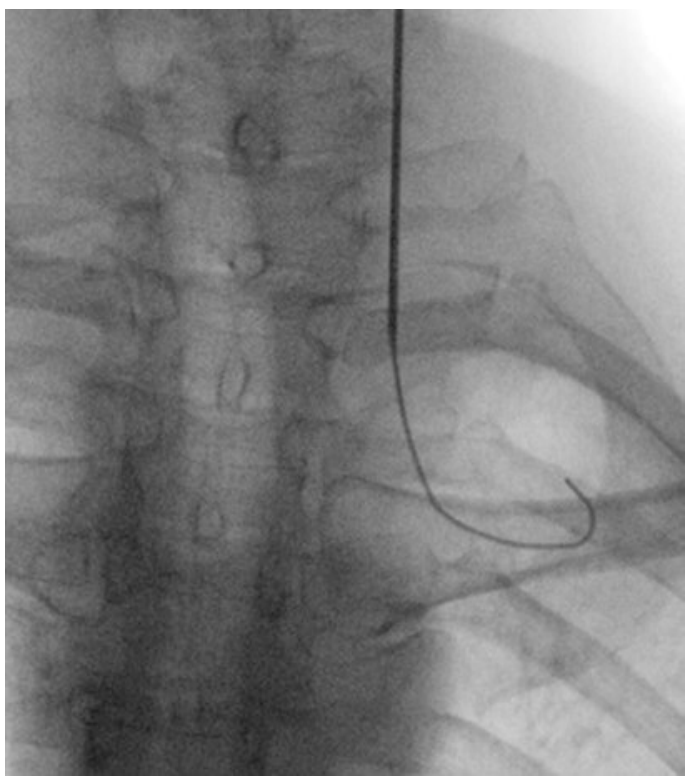

Fig. 7.7 Fluoroscopic image of guidewire advancement to left subclavian (used with permission of S. Hill, Precision Vascular) 


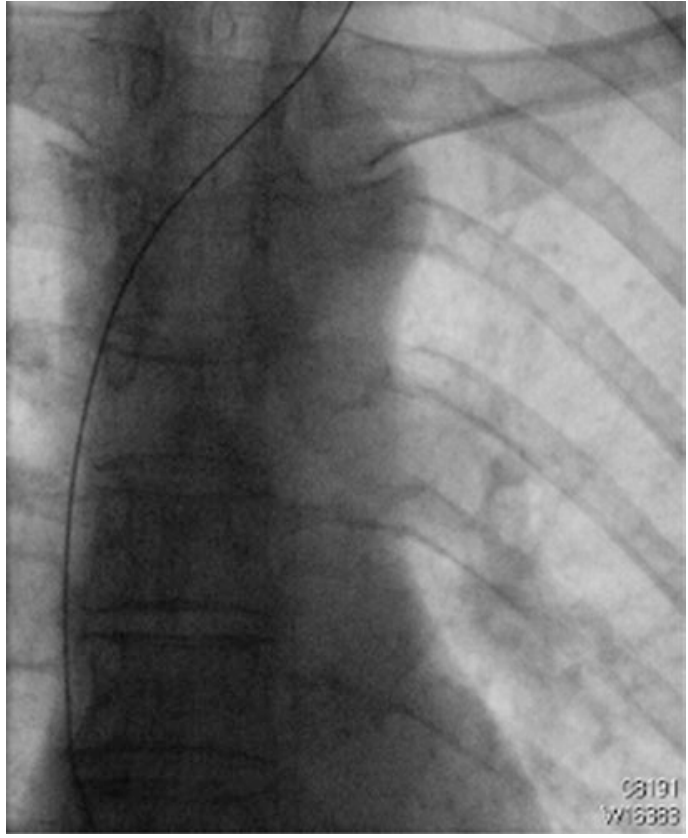

Fig. 7.8 Guidewire in satisfactory position (used with permission of S. Hill, Precision Vascular)

then doubled back on itself at the brachiocephalic junction with the SVC (Fig. 7.9).

The catheter was pulled back and readvanced through the peel-away sheath, and this time it passed into the contralateral veins. An angled hydrophilic guidewire was then used to facilitate passage of the catheter into the correct position.

Though many thousands of catheters are placed routinely without problems, some patients' anatomy present challenges. Having the necessary resources including skills and capacity within radiology is a fundamental necessity and is an inextricable element of vascular access services.

For many years X-ray has been the mainstay and viewed as the gold standard, but now viable alternatives to this are available. The reliability of X-ray is far from absolute. The suggested level of error for clinically significant or major error in radiology ranges from 2 to $20 \%$ (Goddard et al. 2001; Holt and Godard 2012), and a 'real-time' error rate among radiologists of daily practice averages between 3 and 5\% (Brady et al. 2012). Where alternative systems exist that avoid the prescription of ionising radiation, then these systems must unequivocally be considered.

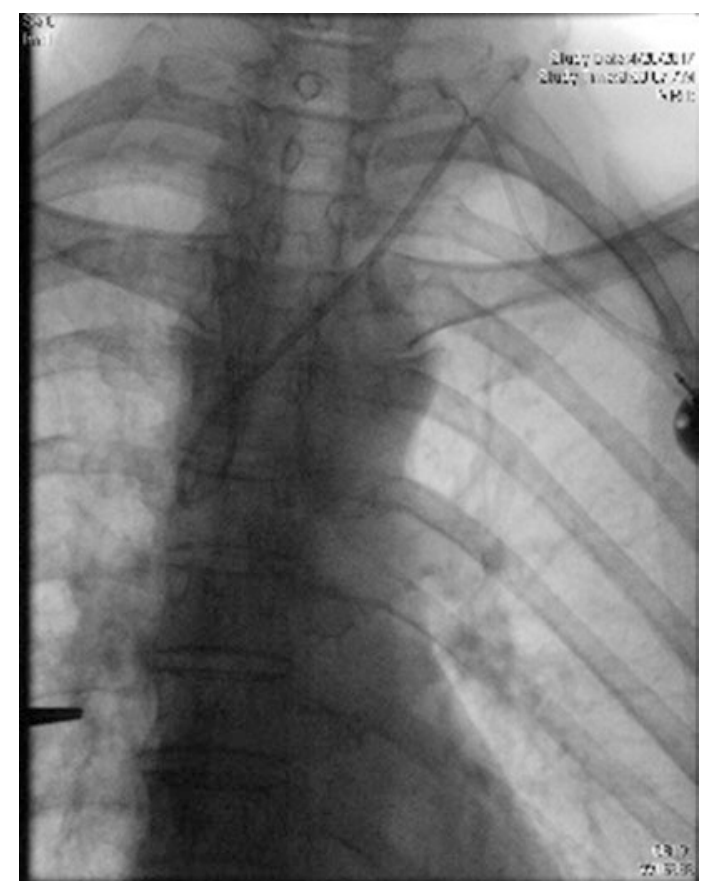

Fig. 7.9 Catheter doubling back into brachiocephalic junction with SVC (used with permission of S. Hill, Precision Vascular)

CVAD performance is directly related to the position of the terminal tip of the catheter (Sansivero 2012). CVAD safety and vein preservation are greatly impacted by tip positioning with device insertion and impacts the ability of the patient to receive prescribed therapy (Moureau et al. 2010). Insertions that are difficult with multiple threading manipulations may result in malpositioned catheters (Fig. 7.10). Suboptimal catheter tip position contributes to catheter dysfunction, thrombosis, vein erosion or continued malposition of the catheter (Eastridge and Lefor 1995; Moureau 2017; Petersen et al. 1999). For optimal upper extremity positioning, the terminal tip should terminate in the lower one-third of the superior vena cava (SVC) near the junction of the SVC and right atrium, where haemodilution rates are highest (Caers et al. 2005; Wuerz et al. 2016). Caers et al. (2005) found that catheter tip locations of CVADs positioned high in the SVC resulted in significant malfunctions. Malpositioned catheters, positioned in a suboptimal location in the SVC or in the adjacent areas, 
are much more likely to develop thrombosis and catheter dysfunction in the form of occlusion (Massmann et al. 2015; Petersen et al. 1999). The conclusions of the research indicated that the terminal tip of CVADs should be positioned as close

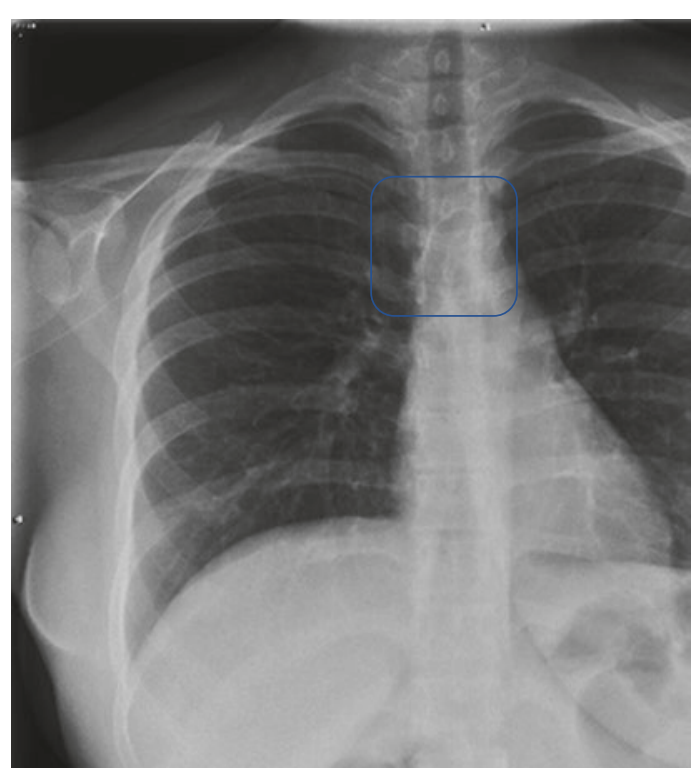

Fig. 7.10 PICC looped in the region of the mediastinum with P-wave elevation. Not distal SVC and no biphasic rhythm (used with permission of S. Hill, Precision Vascular) to the SVC/right atrial junction as possible or slightly inside the right atrium. Multiple factors are involved with correct placement of the CVAD such as skill of the inserter, type of insertion, site selected for insertion and the patients' anatomy (Sansivero 2012).

Correct positioning of a CVAD is verified following the insertion procedure with radiologic confirmation or intraprocedural as with ECG or ultrasound verification. Radiological confirmation, by either chest X-ray or fluoroscopy, expose the patient to radiation and require interpretation on the part of the clinician. Interpretation is based on the anatomical landmarks and the knowledge of the clinician interpreting the results. Landmark determination of CVAD terminal tip precise location is controversial and may be influenced by patient positioning with oblique adjustments, by anatomical variation, complications of pneumothorax or other lung conditions which may impact ability to visualise the catheter tip. Often $\mathrm{X}$-ray views are posterior/anterior and may not capture depth associated with azygous vein malpositioning or accidental arterial insertion (Fig. 7.11). Transoesophageal echocardiogram tip determination is a costly and invasive procedure that is very accurate, but not practical for CVAD insertion position verification.
Fig. 7.11 Accidental arterial PICC insertion (used with permission of S. Hill, Precision Vascular)

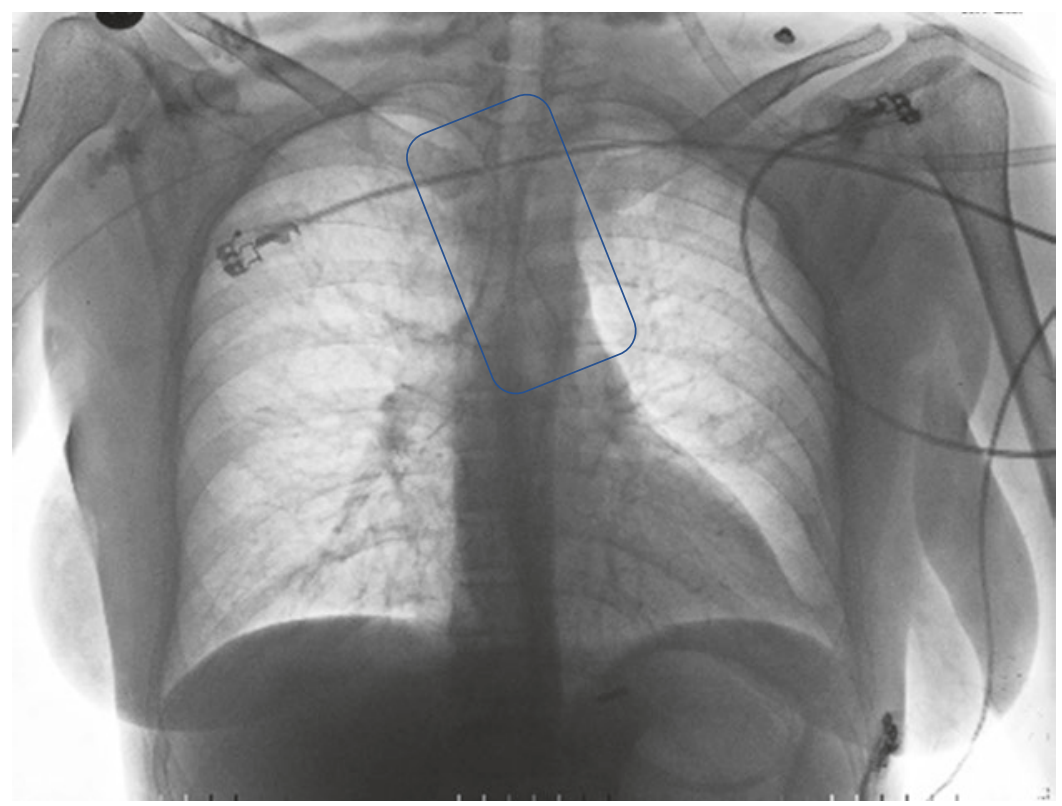


Electrocardiographic intraprocedural positioning and tip confirmation has many advantages. Evidence supports the ECG method as accurate, precise and cost-effective for CVAD terminal tip positioning (Oliver and Jones 2014; Pittiruti et al. 2012; Rossetti et al. 2015; Schummer et al. 2004; Sette et al. 2010; Smith et al. 2010; Wolters et al. 2009). Precise positioning reduces the incidence of thrombotic malfunctions, vessel damage leading to venous thrombosis, arrhythmias, valve damage or other areas of malposition that impact the circulation or cardiac function (Fig. 7.12) (Pittiruti et al. 2011). ECG tip confirmation is performed during insertion using the real-time baseline rhythm connected to a lead on the patient, whilst the wire within the catheter functions as a separate lead (Fig. 7.13). Full description of the technique is included in multiple references (Moureau et al. 2010; Pittiruti et al. 2008, 2011, 2012; Rossetti et al. 2015).

The process for ECG positioning uses impulses from the sinoatrial (SA) node to demonstrate location in respect to the internal catheter. As the catheter advances through the venous system and approaches the SA node, the P-wave, as the first impulse of the pQRS complex, begins
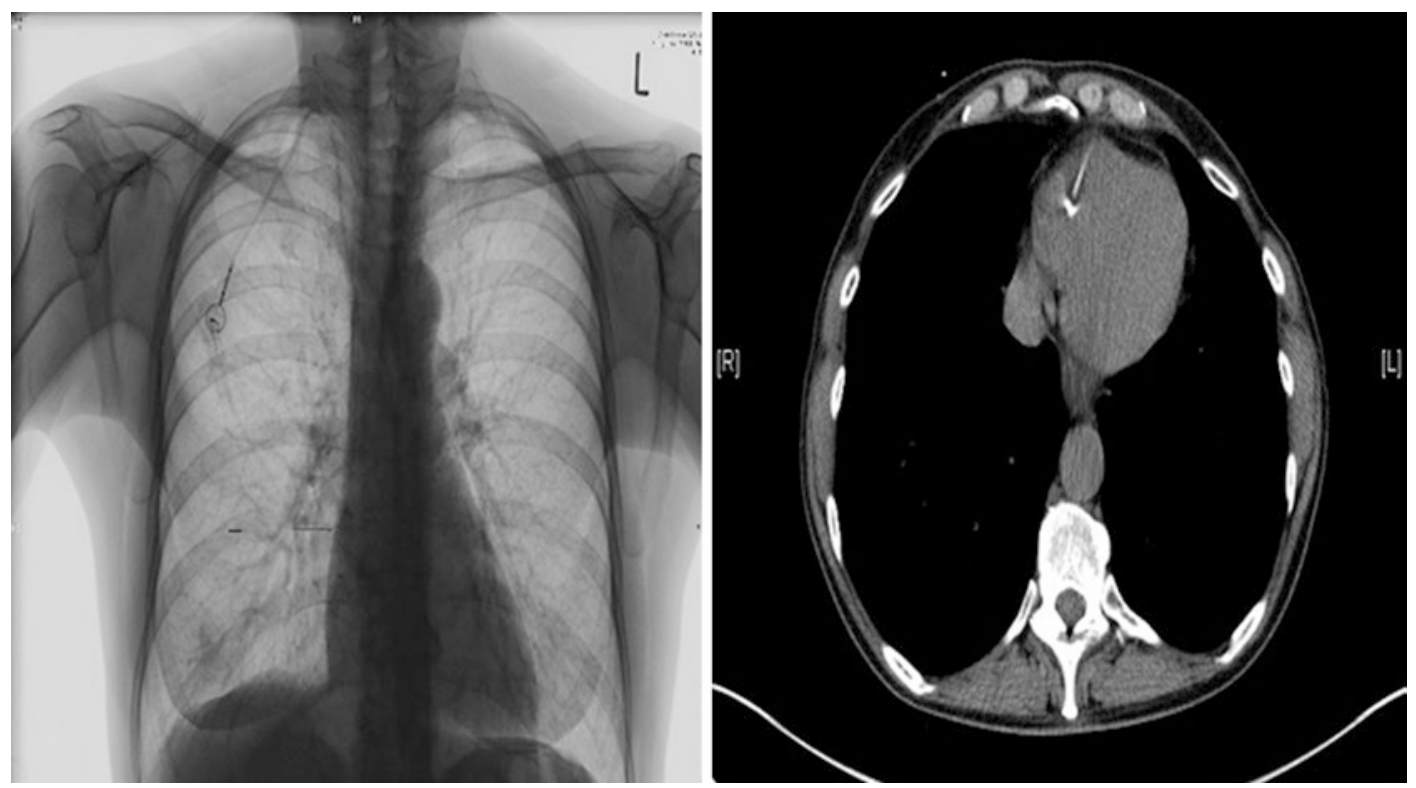

Fig. 7.12 Insertion of port at cavoatrial junction. Computer tomography scan at 20 months within the heart (used with permission of S. Hill, Precision Vascular) to elevate. Maximum intensity is reached as the catheter reaches the cavoatrial junction (CAJ) near the SA node, becoming diphasic with a negative deflection as the catheter passes the node (See Fig. 7.14). Limiting factors of intraprocedural ECG catheter positioning are the absence of a $\mathrm{P}$-wave, as represented in atrial fibrillation and other cardiac rhythms where the P-wave may be indiscernible. Some clinicians have effectively

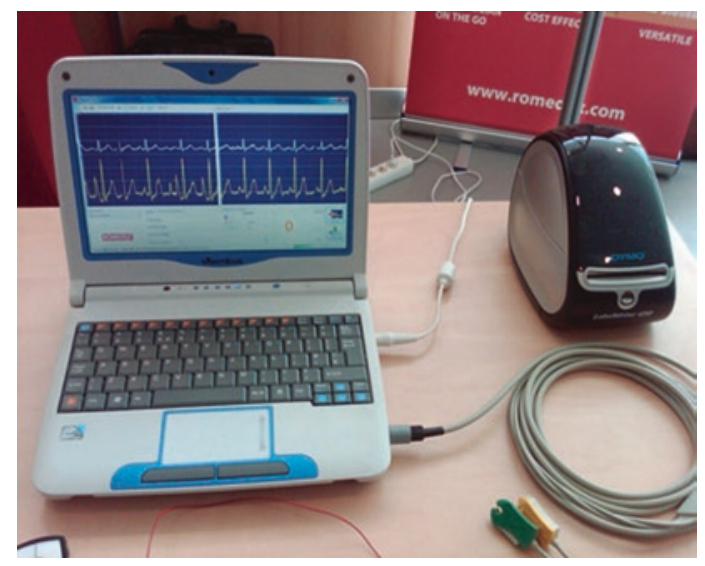

Fig. 7.13 Nautilus system ECG tip positioning system (used with permission of Romedex, Bucharest, Romania) 
Fig. 7.14 Elevation of P-wave (used with permission of Romedex, Bucharest, Romania)

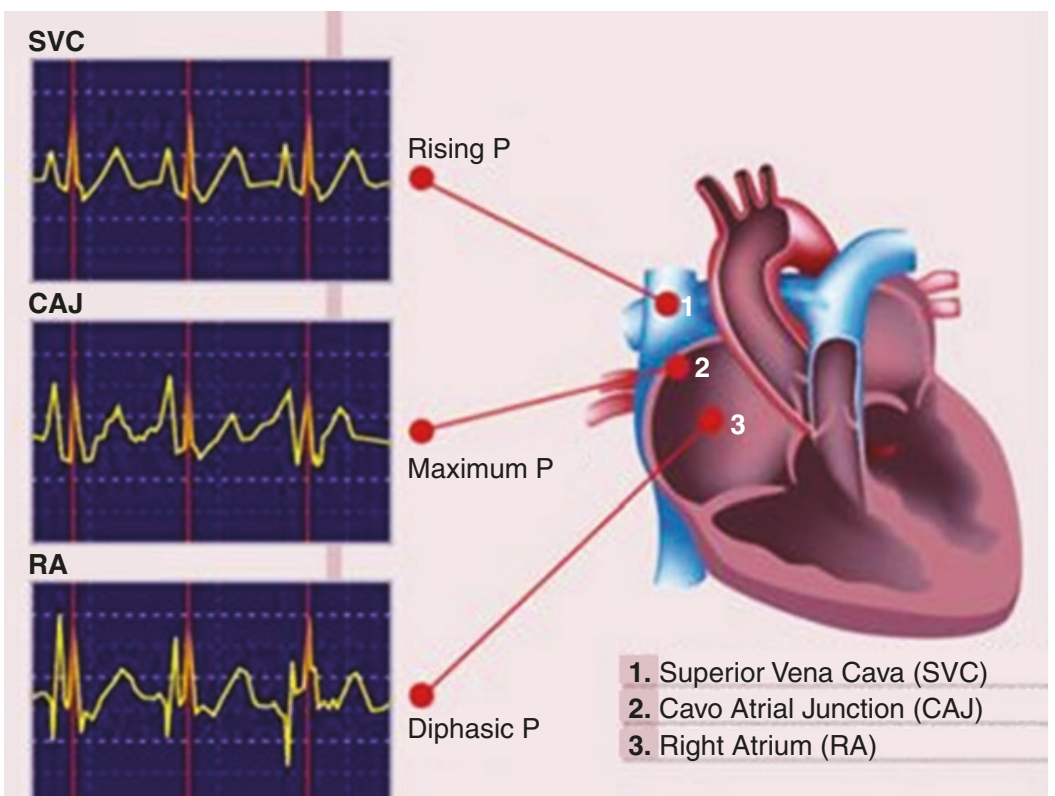

used the ECG system for catheter positioning even in the presence of atrial fibrillation (Pittiruti et al. 2014).

Chest X-ray provides confirmation of the catheter terminal tip position for CVADs whilst facilitating the evaluation of the lungs to rule out pneumothorax. Chest X-ray for CVAD tip verification is based on a one-dimensional view interpreted by the physician (Schuster et al. 2000; Verhey et al. 2008). Interpretation position can be inconsistent with variation from physician to physician; the junction of the SVC and the CAJ junction are not clearly defined on the anteriorposterior chest X-ray (Baldinelli et al. 2015; Pittiruti 2015; Schuster et al. 2000). Chest X-ray is a means to verify tip location but is subject to interpretation and is not precise and is prone (Moureau 2017). Chest X-ray to verify PICC tip position is far from ideal. The time involved in waiting for a chest X-ray to be performed and reported can be extensive, taking $30 \mathrm{~min}$ to multiple hours to complete. Typically, if the location of the terminal tip is incorrect on the X-ray, the clinician returns to the bedside, removes the dressing and repositions the catheter using ANTT. (Moureau 2017). In addition to increasing the risk of contamination, this manipulation can require an additional X-ray to verify whether the repositioning exercise was successful (Gordon 2016). Verification of terminal tip position is a safety requirement which can be satisfied by ECG guidance during placement of the device without the need for X-ray (Moureau 2017).

With ECG guidance, the location of the CVAD is known during the insertion procedure. ECG positioning is both accurate and precise (Pittiruti et al. 2008). ECG positioning is typically not applicable for patients with no discernible P-wave in the QRS complex, with fibrillation or arrhythmias (Pittiruti et al. 2011, 2014). In these situations, a chest X-ray is recommended for verification of the catheter terminal tip.

ECG catheter tip verification is performed by physicians and nurses effectively reducing the time necessary to confirm proper location whilst still in the insertion procedure (Moureau et al. 2010; Pittiruti et al. 2011). The process is easily adapted to the catheter and insertion procedure with equipment provided by multiple companies. In addition, any cardiac monitor can be connected using the lead from the right arm as the catheter lead for intracavitary monitoring. 


\subsection{Steps for Placing ECG- Guided PICC with Guidewire Technique (Moureau et al. 2010)}

1. Attach (three or five lead) monitor to patient (always apply all new leads); determine if the patient is in normal sinus rhythm (NSR) and atrial fibrillation or is dependently paced. If the patient is in NSR, proceed with ECGguided CVAD insertion.

2. Detach the right arm lead from the patient, and attach it to the ECG device or cable. Prepare the cable for use in the sterile procedure (the cable will attach to the guidewire within the catheter).

3. Select the vein and location for CVAD insertion using ultrasound scanning.

4. Measure selected vein to estimated location of caval atrial junction (CAJ).

5. Set up sterile field; prep and drape patient.

6. Don personal protective equipment (PPE).

7. Determine catheter length based on external measurement. Adjust the guidewire to the very distal end of the CVAD but not extended outside the catheter. Flush CVAD with normal saline. Guidewire must be specific for the length of that catheter and marked in advance for intracavitary ECG.

8. Place the sterile ECG cable into the field.

9. Insert the CVAD using ultrasound-guided modified Seldinger technique (MST).

10. Insert CVAD to its intended goal of the distal SVC/CAJ; attach the ECG. Attach alligator cable to the guidewire in the CVAD.

11. Flush the CVAD again with normal saline. The guidewire is acting as an electric conductor of ECG activity.

12. A QRS complex should appear on the monitor with a clearly identifiable P-wave. The $\mathrm{P}$-wave is normal size initially, increasing in amplitude as the CVAD is advanced (Fig. 7.2). Compare P-wave size from initial normal complex to peak level. (Note: Size may vary with QRS complex comparison with P-wave and be larger than QRS. The determinant is positive $\mathrm{P}$-wave change measured to peak with biphasic notch.)
13. When the P-wave is about $3 / 4$ of the full peaked level, approx. 3/4 the size of the QRS, the CVAD tip is in the lower or distal SVC (also known as proximal SVC in relation to the heart).

14. When the P-wave is fully peaked or at the highest amplitude (positive $\mathrm{P}$-wave), it is at the caval atrial junction (SVC/RA).

15. When a small negative wave spike is seen in the P-wave, the tip is in the upper part of the right atrium.

16. When the P-wave becomes biphasic (negative and positive $\mathrm{P}$-wave of the same sizeexpanding beyond the baseline up and down), the CVAD tip is in the middle right atrium. This is known as an atrial spike.

17. If no QRS pattern change is seen during advancement of the catheter, the CVAD has malpositioned in the internal jugular or contralateral in the opposite subclavian vein. Attempts to reposition can be made until the $\mathrm{P}$-wave enlargement is seen on the monitor.

18. Print final strip with $\mathrm{P}$-wave at the same amplitude as QRS to confirm location of the tip. Include this ECG strip as part of the patient's record. (Note: You may see some respiratory variation in the waveform.)

\subsection{Steps for Placing ECG- Guided PICC with Saline- Filled Lumen (Moureau et al. 2010)}

1. Attach (three or five lead) monitor to patient (always apply all new leads); determine if the patient is in normal sinus rhythm (NSR) and atrial fibrillation or is dependently paced. If the patient is in NSR, proceed with ECGguided CVAD insertion.

2. Detach the right arm lead from the patient, and attach it to the ECG device or cable. Prepare the cable for use in the sterile procedure.

3. Select the vein and location for CVAD insertion using ultrasound scanning.

4. Measure selected vein to estimated location of CAJ. 
5. Set up sterile field; prep, and drape patient. Drop ECG cable with saline adapter septum onto the sterile field.

6. Don personal protective equipment (PPE).

7. Determine catheter length based on external measurement. Adjust the guidewire to the very distal end of the CVAD but not extended outside the catheter. Flush CVAD with normal saline. Guidewire must be specific for the length of that catheter and marked in advance for intracavitary ECG.

8. Prefill $20 \mathrm{~cm}^{3}$ syringe with saline and attach a steel needle.

9. Insert the CVAD using ultrasound-guided modified Seldinger technique (MST).

10. Insert CVAD to its intended goal of the distal SVC/CAJ; attach the ECG. Insert needle into ECG saline adapter. Attach alligator clamp to the needle in the CVAD.

11. Flush the CVAD again with normal saline. Saline is acting as an electric conductor of ECG activity.

12. A QRS complex should appear on the monitor with an identifiable $\mathrm{P}$-wave. The $\mathrm{P}$-wave is normal size initially, increasing in amplitude as the CVAD is advanced (Fig. 7.2). Compare $\mathrm{P}$-wave size from initial normal complex to peak level. (Note: Size may vary with QRS complex comparison with P-wave and be larger than QRS. The determinant is positive P-wave change measured to peak with biphasic notch.)

13. When the P-wave is about $3 / 4$ of the full peaked level, approx. 3/4 the size of the QRS, the CVAD tip is in the lower or distal SVC (also known as proximal SVC in relation to the heart).

14. When the P-wave is fully peaked or at the highest amplitude (positive $\mathrm{P}$-wave), it is at the CAJ (SVC/RA).

15. When a small negative wave spike is seen in the P-wave, the tip is in the upper part of the right atrium.

16. When the P-wave becomes biphasic (negative and positive $\mathrm{P}$-wave of the same sizeexpanding beyond the baseline up and down), the CVAD tip is in the middle right atrium. This is known as an atrial spike.
17. If no QRS pattern change is seen during advancement of the catheter, the CVAD has malpositioned in the internal jugular or contralateral in the opposite subclavian vein. Attempts to reposition can be made until the $\mathrm{P}$-wave enlargement is seen on the monitor.

18. Print final strip with P-wave at the same amplitude as QRS to confirm location of the tip. Include this ECG strip as part of the patient's record. (Note: You may see some respiratory variation in the waveform.)

19. Print final strip with P-wave at peak amplitude as QRS to confirm location of the tip. Include this ECG strip as part of the patient's record. (Note: You may see some respiratory variation in the waveform.)

\subsection{Tip Movement}

The catheter tip is exposed to many mechanical forces, exerted by the internal mechanical functions of the human body that can affect and alter the internal position, namely, the expansion and deflation of the lungs during the respiratory cycle and the pulsation of the heart and arterial structures. Vesely (2003) confirms the continual movement of the catheter tip from cardiac pulsation can lead to thrombosis development (Vesely 2003). The position of the patient will also affect the position of the catheter tip whether sitting, standing, supine or when considering PICCs if the arm is abducted or adducted. Foaurer and Alonzo (2000) measured the effect of moving the arm from an abducted to adducted position in 61 patients and found that 43 patients move caudally (lower/towards the heart), 7 moved cephalad (higher or superiorly) and 3 did not change position; the authors summarised that $58 \%$ of PICCs move $2 \mathrm{~cm}$ or more (Foaurer and Alonzo 2000). Hostetter et al. (2010) confirm that movement of catheter tip position is reported to be $2-3 \mathrm{~cm}$; a correctly CAJ placed catheter has the potential to retract into the SVC and advance into the RA. Considering such variation occurs when the arm is abducted, bringing the arm away from the $90 \%$ extended, abducted position prior 


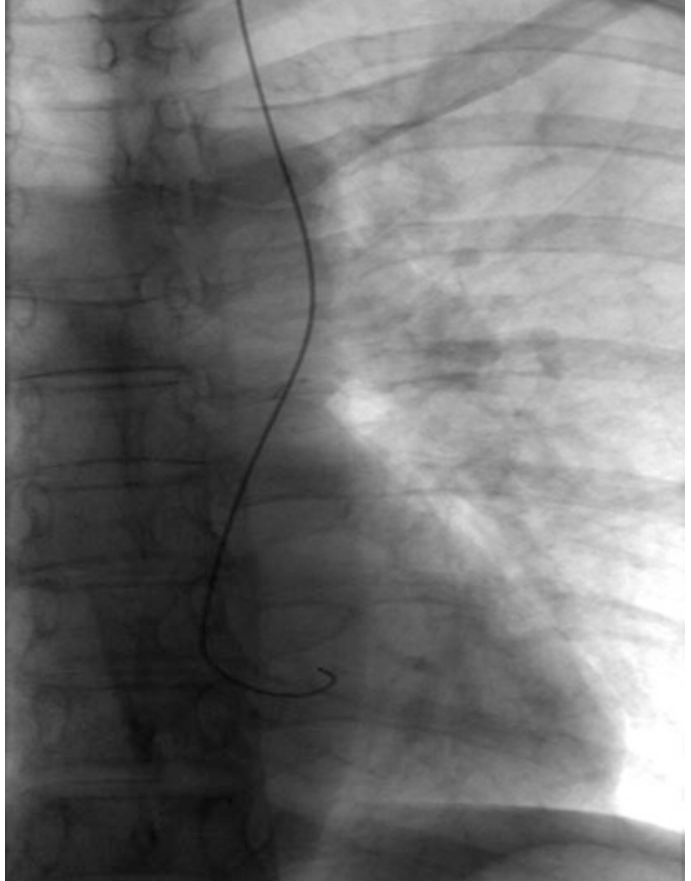

Fig. 7.15 Left internal jugular placement with incidental finding of left-sided SVC (used with permission of S. Hill, Precision Vascular)

to confirming tip position, more in line with the body's natural position may reduce migration associated with arm abduction.

Anatomical deviations may be present such as scoliosis, dextrocardia, left-sided SVC (Fig. 7.15) or iatrogenic abnormalities such as pneumonectomy (Fig. 7.16). Catheter tip position is not static like the snapshot image that an X-ray provides, which portrays a moment in time of the catheter tip position. Furthermore, this snapshot is often not a typical representation of the catheter tip position. It is standard practice to ask the patient to take a deep breath and hold the breath when the X-ray is being taken. The deep inhalation and breath holding force the diaphragm low, lowering the position of the heart within the thorax and altering the tip position of the catheter. Similarly, the position of the patient can affect the position of the catheter tip.

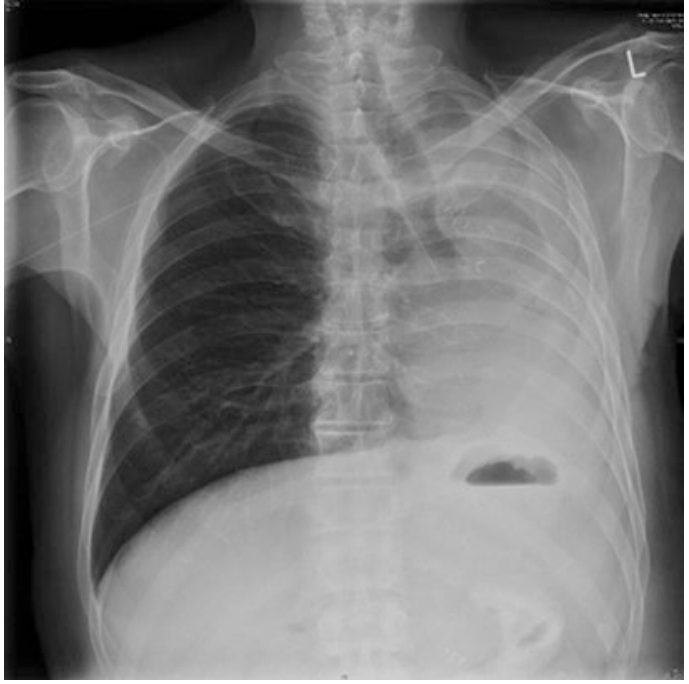

Fig. 7.16 Left pneumonectomy, right-sided PICC insertion; the PICC extended across the mediastinum to reach optimal position (used with permission of S. Hill, Precision Vascular)

\subsection{Thrombosis}

The position of the catheter tip can influence the risk of VAD-related thrombosis. The actual position of the catheter tip can influence the likelihood of the catheter developing a thrombus. If the position of the catheter is too long and too short, abuts the wall of a vessel or is positioned optimally, all can influence the risk of thrombosis. An older study looking at right atrial thrombus on 48 patients with tunnelled CVC in situ, tip positions were in the superior vena cava and the right atrium (RA). Thrombus was found in $12.5 \%$ of cases by follow-up with transoesophageal echocardiograph, all of the thromboses were identified in the RA group, and all were asymptomatic (Gilon et al. 1998). Other studies have found higher incidence of thrombus in the SVC (Vesely 2003).

A larger randomised controlled study looked at this issue. Figure 7.17 is from a study looking at $428 \mathrm{CVC}$ insertions, followed up with linograms, venograms and Doppler ultrasounds. What the study showed was that the relative position of 


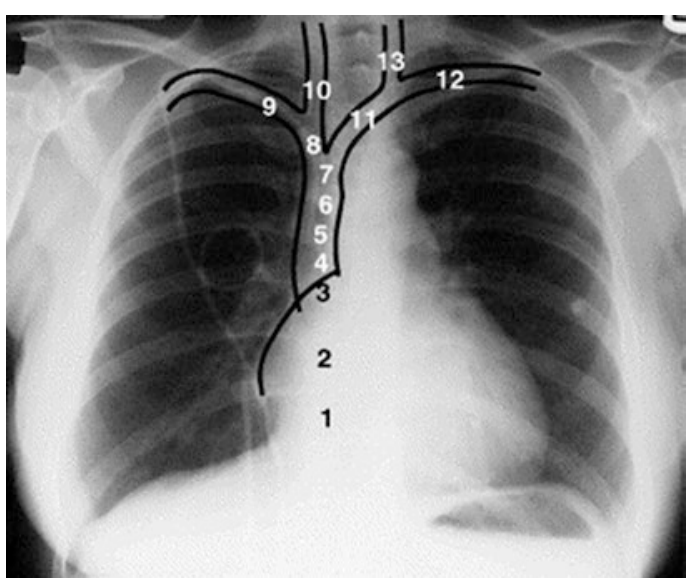

Fig. 7.17 Radiologic anatomy and tip position for CVADs (Cadman et al. 2004)

the catheter tip was directly proportional to the risk of thrombosis development. For instance, on the image, numbers 3 and 4 indicate distal SVC/ cavoatrial junction, and for the catheters with the tips positioned in this zone, the thrombosis rates were 2.6\%; in the middle third of the SVC, 5.3\%; and in the proximal position (proximal SVC or thoracic inlet veins), $41 \%$ resulted in thrombosis, an increase of 16 times the risk of those catheters placed in a distal position. The reason the authors gave for increased risk of short left-sided catheters was the catheter abutting the wall of the SVC, causing mechanical damage and also the potential for chemical damage from cytotoxic drugs inducing thrombotic changes (Cadman et al. 2004; Vesely 2003). Hallam et al. (2016) also include device selection based on nutritional solutions containing final concentrations exceeding $10 \%$ dextrose and/or 5\% protein and explain that these also should be administered via a central venous catheter with tip placement in the superior vena cava. DeChicco et al. (2007) also identify that thrombosis is greater in the proximal SVC versus lower SVC and RA.

The study by Cadman and colleagues shows significant associations between thrombosis and females (twice the risk of males), left-sided insertions and proximal tip position. Catheter tip in the proximal third of the SVC and above was 16 times more likely to develop venous thrombosis. Women were twice more likely to develop throm- bosis than men. Left-sided CVAD insertions may lead to damage as stiff dilators/introducers are passed through the brachiocephalic vein to the SVC (Vesely 2003).

Hostetter et al. (2010) points out that the presence of thrombosis may lead to infection. The apparent symbiotic relationship between thrombosis and infections has been avidly discussed within the field of vascular access. Theorists and clinicians suggest that the composites of a thrombus encourage the growth of pathogenic microbes and conversely the presence of infection releases coagulative processes that increase the patient to catheter-related thrombosis (Mehall et al. 2002; Raad and Bodey 1992; Ryder 2001).

Right atrial thrombus - Some studies have looked at the development of thrombosis in the heart. Vesely (2003) explains that catheters placed within the heart may lead to intracardiac thrombosis but points out that some studies (Kung et al. 2001) show that the placement of the catheter tip in the SVC did not prevent the formation of right atrial thrombus (RAT). Dreyer and Bingham (2005) investigating the right atrial thrombosis related to haemodialysis catheters reporting from 22 cases of RAT report that a $33 \%$ mortality exists if RAT is associated with infection versus $14 \%$ without infection (Dreyer and Bingham 2005). In terms of management of RAT, the authors suggest that thrombectomy has been associated with lower mortality than conservative management with anticoagulants and antibiotics. A strategy adopted by some includes a thrombosis less than $2 \mathrm{~cm}$ being managed conservatively (anticoagulants for 6 months) with repeated transoesophageal echocardiograph and catheter removal, larger than $2 \mathrm{~cm}$, especially if infected, thrombectomy with antibiotics and anticoagulants (Dreyer and Bingham 2005). However, the authors concede that the optimal management of haemodialysis RAT is unknown.

Current practice in many institutions is still for a post-procedural chest X-ray to be used to verify the tip position of the catheters following insertion, for services inserting by the bedside, or in a designated procedure room without ECG guidance or X-ray screening. This can result in patient delays until the X-ray has been undertaken and 
verified. The limitations of this approach are that there is limited intraprocedural awareness of catheter tip position from the point of insertion to the landing zone.

Rates of malposition vary depending upon experience and type of insertion. Ibrahim (2012) identifies a bedside malposition rate for nontunnelled CVCs of $14 \%$, with $55 \%$ of these tips being misplaced into the RA and $14 \%$ into the left brachiocephalic vein. Other studies have shown that CVCs have 6.7\% malposition rate (Roldan and Paniagua 2015) and PICC malposition rates of $9.3 \%$ both without guidance technology, confirmed by Hill (2012) whose combined PICC and CVC non-guided malposition rates were reported at $8 \%$. Some studies show much higher malposition rates, up to $41 \%$ for central placements, including tips placed in the proximal third of SVC, including thoracic inlets, and PICC malposition rates up to $63 \%$ (Johnston et al. 2013). In cases of malposition, the catheter may require manipulation under fluoroscopy, retracting if too long or removing and replacement (Fig. 7.18). Manipulation of a catheter depends upon the availability of the fluoroscopy suite and radiologist or suitably trained clinician. If fluoroscopy

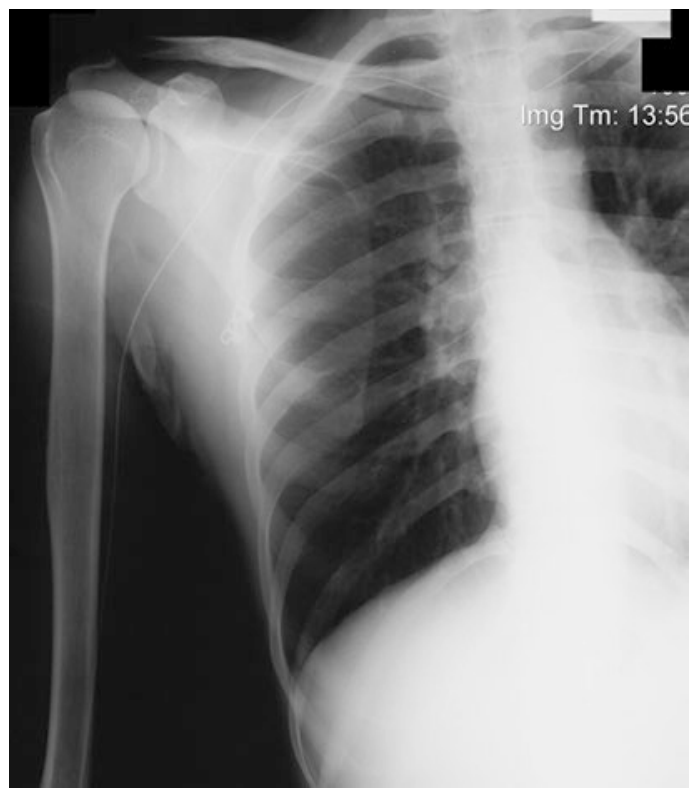

Fig. 7.18 Contralateral malposition with oblique (used with permission of N. Moureau, PICC Excellence) is in high demand, immediate access may not be possible, increasing patient treatment delay and increasing costs attributed to the use of fluoroscopy. The additional interventions required to rectify misplaced catheters cause increased patient anxiety/distress and increase the risk of infective and mechanical complications. The additional interventions also decrease the efficacy and cost efficiency of the vascular access (VA) team.

Dialysis - Tal et al. (2013) explain dialysis catheter guidelines recommend the catheter to be placed into the RA. The authors also advocate that not only the tip of the catheter but the 'functional tip' of the catheter should be placed in the RA whilst ensuring that the catheter does not touch the atrial floor as to avoid mural thrombus, arrhythmias and erosion. The authors describe the function tip as the portion of the catheter from the most proximal side hole to the catheter tip. SVC placement of dialysis catheters can lead to higher recirculation rates, damage to the vessel wall, stenosis and catheter occlusion ( $\mathrm{Tal}$ et al. 2013). Mandolfo et al. (2002) identifies that dialysis catheter tips placed at the cavoatrial junction benefit from higher blood flow. Equally Vesely (2003) suggests that stagnation of blood within the RA may lead to thrombus formation.

The national kidney foundation clinical guidelines advocate:

The catheter tip should be adjusted to the level of the caval atrial junction or into the right atrium to ensure optimal blood flow. (Atrial positioning is only recommended for catheters composed of soft compliant material, such as silicone) (Opinion) NKF-K/DOQI (2001)

The CAJ cannot be definitively identified on an X-ray; rather the position of the CAJ is inferred by looking at other visible anatomical structures.

CXR-X-ray images are also subject to an element of magnification; here is an example in practice. The magnification associated with fluoroscopy is illustrated by placing two identicalsized coins, one on the anterior chest wall and the second coin level with the patient's back/spine. The extent to which magnification affects the image is dependent upon the distance the inten- 


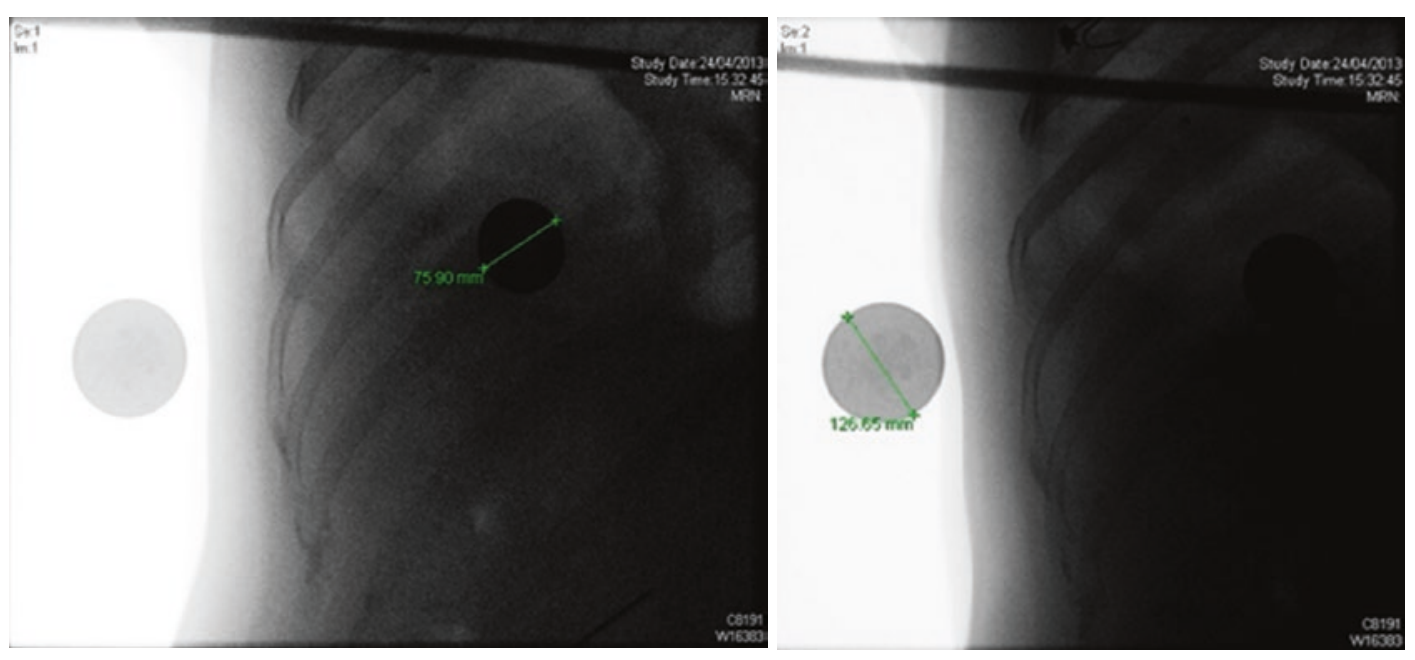

Figs. 7.19 and 7.20 Lateral view with magnification of the chest using coin perspective (used with permission of S. Hill, Precision Vascular)

sifier is from the patient. The patient's physical size can affect this, i.e. an increase in patient size results in increased distance from intensifier to plate resulting in more magnification. This is illustrated in Figs. 7.19 and 7.20 using the same size coins, one placed level with the anterior surface of the chest and the second with the patient's back. The coin placed posteriorly appears significantly larger (126.65 $\mathrm{mm})$ versus the anteriorly placed coin measuring 75.90 , which illustrates the increasing magnification of internal anatomy the closer it is to the image receptor.

Parallax-Vesely (2003) describes that the phenomenon of parallax can be an influential in determining catheter tip position.

Parallax is defined as:

the position of the image on each emulsion of dual emulsion film; it is accentuated by tube-angled $\mathrm{x}$-ray techniques.

Miller-Keane (2003)

The objects in the lateral view are affected depending upon their relative position to each other and the angle at which they are viewed. Place a finger in front of you and view it with one eye closed then alternate by closing that eye and opening the other, the differing visual perspectives is an example of how the parallax effect works.
Considering the effects in practice, when viewing tip position on X-ray, it is important to select an anatomical structure closest to the catheter tip position to help to determine its position. Parallax may have more effect the further away the structure is from the point of interest; for instance, determining tip position by looking at the ribs and selecting anatomy in different anatomical planes can lead to error (Aslamy et al. 1998). Ryu et al. (2007) points out that parallax is greater and more variable with portable anterior-posterior CXR, such as in intensive care and operating theatres (Ryu et al. 2007).

Aslamy et al. (1998) suggested the tracheobronchial angle was the best radiographic landmark to determine the upper margin of the SVC. The authors determined these conclusions by studying 42 patients who had undergone magnetic resonance imaging scans (Aslamy et al. 1998). Hostetter et al. (2010) explain the carina later replaced this landmark because the structure can be identified more clearly. Baskin and colleagues confirmed this by examining $100 \mathrm{CT}$ scans of patients from the ages of 12 to 28 years and found no association between age and any other parameters. The authors go on to explain that the cavoatrial junction lies lower than commonly believed and suggest that two verte- 
bral bodies below the carina (with the intervertebral disc included) is a reliable estimate of the position of the cavoatrial junction (Baskin et al. 2008). Aslamy et al. (1998) importantly identified that the right superior heart border that is commonly used to determine catheter tip location, in $38 \%$ of cases, was formed by the left atrium.

Movement of tip position-Catheter tips are prone to movement; the still image of a radiographic X-ray detracts from this notion. The catheter tip is subjected to a range of dynamic movements from cardiac pulsation and lungs/diaphragmatic movement, which all contribute to the perpetual motion of the catheter tip (Mandolfo et al. 2002). The position of the patient can affect tip, whether standing, holding their breath for an $\mathrm{X}$-ray, abducting or adducting their arm position (Fig. 7.21). The catheter with the most potential for internal catheter movement is a PICC. If the arm is abducted away from the body, this can cause downward internal movement of the PICC towards the heart of up to $2 \mathrm{~cm}$; right-sided

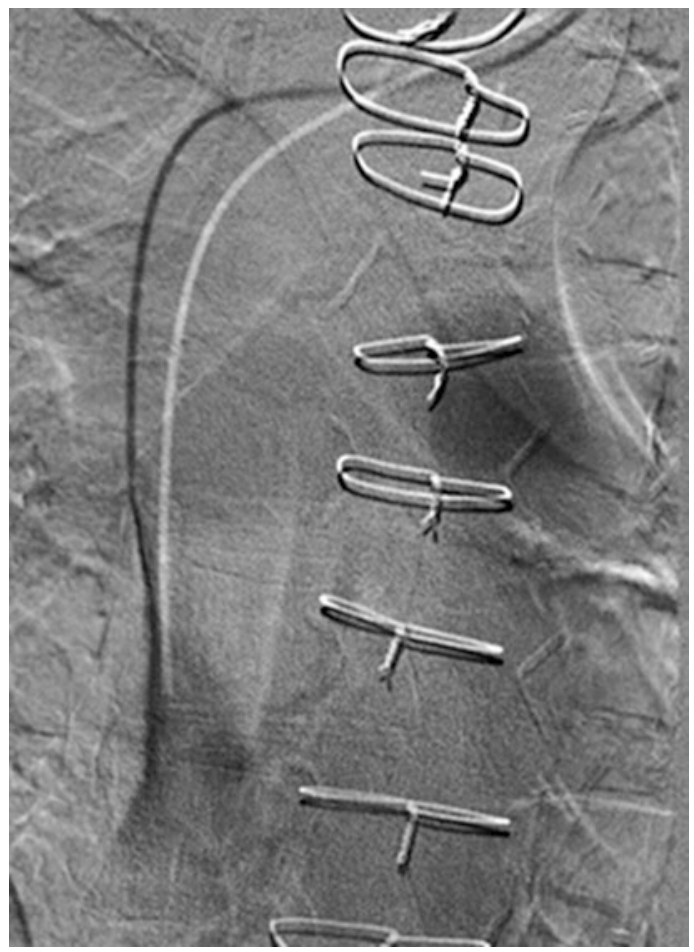

Fig. 7.21 PICC tip movement during high-pressure injection (used with permission of S. Hill, Precision Vascular)
PICCs have higher rates of this type of movement vs the left (Aslamy et al. 1998).

Tunnelled CVCs and implanted ports can have different anatomical sites where they can be situated. Typically, they are placed on the anterior chest wall, which has an immediate benefit over PICCs in the stakes of internal movement, as they are not influenced to the same extent by the arm movement as the peripherally inserted counterparts. However, what is commonly appreciated by vascular access clinicians, especially those working in fluoroscopy, is that significant catheter tip movement is associated with patient position changing from supine to standing. Gibson and Bodenham (2013) point out that this movement is due to gravity, and as the abdominal contents move downwards, so do the diaphragm and the mediastinum, inadvertently causing an upward movement of the catheter tip. The authors also point that this is exacerbated in obese patients. I would add that the most profound movement observed of long-term catheters is in ladies who have an increased body mass index, who have large breasts and where the CVADs are being placed on the left. The movement for left-sided long-term devices appears to be more significant compared with those on the right, one way of counteracting this is to stabilise the chest wall in a position that simulates a supine position. This can be done by placing downward traction of the upper chest wall with adhesive drapes or using sensitive tapes; this simulation of supine chest wall/breast position limits, but does not eradicate, the tip movement from the patient moving from supine to the standing position. Large breast tissue has been documented in several studies as an influential factor in catheter tip placement (Gibson and Bodenham 2013; Cadman et al. 2004; Vesely 2003).

$\mathrm{X}$-ray outcomes are also subject to the skill of the radiographer and the presentation of the patient, obesity, patient position, if the patient needs a mobile X-ray, or there may be other factor such as Harrington rods used for spinal support that may obscure the view of the catheter tip. The objective of a normal posterior (PA) anterior X-ray is to provide a balanced field of view to enable examination of the skeletal structures, organs and 
tissues. The objective of an X-ray post-CVAD insertion is to primarily identify the tip position, but at the same time, the clinician will ensure there are no kinks or loops in the catheter. There may be occasion that the purpose of the X-ray is dual fold, for the identification of tip position and to view the thoracic anatomical structures. The radiation prescription for a standard X-ray film may not necessarily be the dose needed to adequately view the tip position of a smallersized vascular device such as a PICC. Obtaining adequate exposure to identify the catheter tip is essential, or repeated X-rays may be needed. If a repeat CXR is required, a lateral view may provide an alternative angle if the tip is obscured on a PA or X-ray can be coned in to focus on the mediastinal area, which will reduce the radiation dose to the surrounding thoracic anatomy.

\subsection{Conclusion}

Tip position continues to be an emotive and controvertial subject. Clinicians must be aware of the range of movements a CVAD catheter tip is subjected to when the patient changes positions, from supine to standing the abdominal contents drop causing upward movement of the catheter tip (Vesely 2003), and the movement of PICCs particularly with abduction of the arm, and consider these potential movements when finalising catheter tip position. The revolution in catheter tip navigation and confirmation is now in full flow; the paradigm shift from radiological confirmation to ECG-based technology is now being reflected practice across the globe. The accuracy, safety benefits, elimination of radiation exposure, improved patient experience and vascular access service efficiency no longer allow X-ray confirmation to be the 'gold standard'.

\section{Case Study}

The patient was a 60-year-old female with a diagnosis of colonic adenocarcinoma, who had received a previous tunnelled CVC via the right internal jugular side that had been removed due to infection during a previous cycle of intravenous anticancer therapy. The patient attended for a pre-assessment for an implanted port and underwent all the necessary checks, including a review of the patient history, assessment of the right anterolateral upper chest wall (intended port implantation site), blood tests and ultrasound review of the relevant vasculature, taking time to talk through the procedure with the patient and provide the supportive literature about the procedure. The assessment was completed, and no concerns were noted about venous or other anatomy, and blood tests were obtained as per hospital protocol. During the procedure, ultrasound-guided cannulation was performed routinely, but the clinician could not pass guidewire; a more experienced operator attended who experienced same issue. As the procedure was undertaken in a dedicated procedure room intended to be placed under ECG guidance, it was decided that the procedure should be undertaken in the X-ray department under fluoroscopy and was rescheduled for another day, as not available that day. A left-sided internal jugular approach was planned. The ultrasound assessment showed an interesting image of duplicated left internal jugular veins. Passing the ultrasound from higher up the neck to the base, superiorly to inferiorly, the veins were one vessel (higher in the neck) which then split into two as they both then led separately to form part of the brachiocephalic vein. The vein positioned more anteriorly was cannulated under ultrasound, being more superficial and the larger of the two vessels (Fig. 7.6). 


\section{Case Study}

A call was received from a radiologist to the vascular access team. The radiologist explained there was a patient who had undergone a CT scan and noticed that the catheter tip was in the ventricle. With great concern, the team evaluated the terminal tip position. The patient had also had a CXR that showed the catheter tip well into the heart. This implanted port had been in for 20 months. What had gone wrong, and how had this been missed? The port chamber was in its original position and had not slipped out of position; the catheter was still securely attached to the injectable reservoir and had not fractured and migrated. Upon closer investigation of this case, it became apparent that the port position hadn't changed but rather the internal anatomy. Unfortunately, over the 20 months from insertion, the patient had slowly developed bulky abdominal disease leading to upward movement of the diaphragm, resulting in the downward displacement of the catheter tip. Position was corrected by port revision with tip placed in the distal SVC rather than at the CAJ to avoid continued deterioration of the patient. Documentation was established in the medical record to alert medical providers of the changes in anatomy and impact on the port if the abdominal mass is removed.

\section{Case Study}

A patient required 6 weeks of antibiotic treatment for osteomyelitis. It was determined that a PICC would be the best device for the treatment duration and medication. The patient was positioned and measured for a placement length estimate. The PICC was placed by a trained specialist. The PICC was inserted with maximum sterile barriers, ultrasound guidance and ECG. The monitor and leads were prepared in advance and connected in a sterile fashion during the procedure. Prior to the procedure, the baseline EKG was established. During the procedure, the internal EKG rhythm demonstrated advancement of the PICC as the catheter entered the SVC. The P-wave continued to increase in amplitude as the catheter approached the cavoatrial junction (CAJ) reaching maximum. No biphasic or downward deflection of the P-wave noted. PICC was aspirated to confirm blood return, flushed with normal saline, secured and the procedure completed.

\section{Summary of Key Points}

1. The aim for vascular access clinicians is to place the catheter tip in the optimal location.

2. The implications of catheter malposition can contribute to increased morbidity.

3. The evolution of radiation-based methods to confirm catheter tip position can no longer claim the title of the 'gold standard' in practice.

\section{References}

Alexandrou E, Ramjan L, Spencer T, Frost S, Salamonson Y, Davidson P, Hillman K. The use of midline catheters in the adult acute care setting-clinical implications and recommendations for practice. J Assoc Vasc Access. 2011;16(1):35-41.

Aslamy Z, Dewald CL, Heffner JE. MRI of central venous anatomy. Implications for central venous catheter insertion. Chest. 1998;114:820-6.

anatomy: implications for central venous catheter insertion. Chest. 1998;114(3):820-6.

Baldinelli F, Capozzoli G, Pedrazzoli R, Marzano N. Evaluation of the correct position of peripherally inserted central catheters: anatomical landmark vs. electrocardiographic technique. J Vasc Access. 2015;16(5):394-8. https://doi.org/10.5301/ jva.5000431.

Baskin KM, Jimenez RM, Cahill AM, Jawad AF, Towbin RB. Cavoatrial junction and central venous anatomy: 
implications for central venous access tip position. J Vasc Interv Radiol. 2008;19(3):359-65.

Bertoglio S, Van Boxtel T, Goossens GA, Dougherty L, Furtwangler R, Lennan E, Pittiruti M, Sjovall K, Stas M. Improving outcomes of short peripheral vascular access in oncology and chemotherapy administration. London: SAGE Publications; 2017.

Bestman E, Creese R. Waller-pioneer of electrocardiography. Br Heart J. 1979;42(1):61-4.

Brady A, Laoide RÓ, McCarthy P, McDermott R. Discrepancy and error in radiology: concepts, causes and consequences. Ulster Med J. 2012; 81(1):3.

Burdon-Sanderson JS, Page FJM. II. Experimental results relating to the rhythmical and excitatory motions of the ventricle of the heart of the frog, and of the electrical phenomena which accompany them. Proc R Soc Lond. 1878;27(185-189):410-4.

Cadman A, Lawrance J, Fitzsimmons L, Spencer-Shaw A, Swindell R. To clot or not to clot? That is the question in central venous catheters. Clin Radiol. 2004;59(4):349-55.

Caers J, Fontaine C, Vinh-Hung V, De Mey J, Ponnet $\mathrm{G}$, Oost C, et al. Catheter tip position as a risk factor for thrombosis associated with the use of subcutaneous infusion ports. Support Care Cancer. 2005;13(5):325-31.

Campisi C, Biffi R, Pittiruti M. Catheter-related central venous thrombosis: the development of a nationwide consensus paper in Italy. J Assoc Vasc Access. 2007;12(1):38-46.

Chopra V, Flanders SA, Saint S, Woller SC, O'Grady NP, Safdar N, Trerotola SO, Saran R, Moureau N, Wiseman S, Pittiruti M, Akl EA, Lee AY, Courey A, Swaminathan L, Ledonne J, Becker C, Krein SL, Bernstein SJ, Michigan Appropriateness Guide for Intravenouse Catheters Panel. The Michigan Appropriateness Guide for Intravenous Catheters (MAGIC): results from a multispecialty panel using the RAND/UCLA appropriateness method. Ann Intern Med. 2015;163:S1-40.

Cushny AR, Edmunds CW. Paroxysmal irregularity of the heart and auricular fibrillation. In: Bulloch W, editor. Studies in pathology. Written by alumni to celebrate the quatercentenary of the University of Aberdeen and the quarter-centenary of the chair of pathology therein. Aberdeen: Aberdeen University; 1906. p. 95.

DeChicco R, Seinder D, Brun C, Steiger E, Stafford J, Lopez R. Tip position of long-term central venous access devices used for parenteral nutrition. J Parenter Enter Nutr. 2007;31(5):382-7.

Dreyer G, Bingham C. Right atrial thrombus as a complication of a temporary haemodialysis catheter-a potentially avoidable complication. Nephrol Dial Transplant. 2005;20(2):474-5.

Duran-Gehring PE, Guirgis FW, McKee KC, Goggans S, Tran H, Kalynych CJ, Wears RL. The bubble study: ultrasound confirmation of central venous catheter placement. Am J Emerg Med. 2015;33(3):315-9.
Eastridge B, Lefor A. Complications of indwelling venous access devices in cancer patients. J Clin Oncol. 1995;13(1):233-8.

Einthoven W. Ueber die Form des menschlichen Electrocardiogramms (Using an improved electrometer and a correction formula developed independently of Burch, distinguishes five deflections which he names P, Q, R, S and T). Pflügers Arch Eur J Physiol. 1895;60(3):101-23.

Farlex. Farlex Partner Medical Dictionary. Splinting; 2012.

Foaurer AR, Alonzo M. Change in peripherally inserted central catheter tip position with abduction and adduction of the upper extremity. J Vasc Radiol. 2000;11(10):1325-8.

Gibson F, Bodenham A. Misplaced central venous catheters: applied anatomy and practical management. $\mathrm{Br}$ J Anaesth. 2013;110(3):333-46.

Gilon D, Schechter D, Rein AJ, Gimmon Z, Or R, Rozenman Y, Slavin S, Gotsman MS, Nagler A. Right atrial thrombi are related to indwelling central venous catheter position: insights into time course and possible mechanism of formation. Am Heart J. 1998;135(3):457-62.

Goddard P, Leslie A, Jones A, Wakeley C, Kabala J. Error in radiology. Br J Radiol. 2001;74(886):949-51.

Gordon S. Bedside chest radiographs and how ambiguous peripherally inserted central catheter tips happen: a case report. J Assoc Vasc Access. 2016;21(4): 237-41.

Gorski L, Hadaway L, Hagle M, McGoldrick M, Orr M, Doellman D. Infusion therapy: standards of practice. J Infus Nurs. 2016a;39(Suppl 1):S1-S159.

Gorski L, Hadaway L, Hagle M, McGoldrick M, Orr M, Doellman D. INS infusion therapy: standards of practice (supplement 1). J Infus Nurs. 2016b;39:S1-S159.

Hadaway LC. I. V. infiltration: not just a peripheral problem. Nurs Manag. 2000;31(11):25-32.

Hallam C, Weston V, Denton A, Hill S, Bodenham A, Dunn H, Jackson T. Development of the UK Vessel Health and Preservation (VHP) framework: a multi-organisational collaborative. J Infect Prev. 2016;17:65-72.

Hellerstein HK, Pritchard WH, Lewis RL. Recording of intracavity potentials through a single lumen, saline filled cardiac catheter. Proc Soc Exp Biol Med. 1949;71(1):58-61.

Hill S. VasoNova Arrow VPSTM technology evaluation. Hospital Pharmacy Europe; 2012.

Holt J, Godard P. Discrepancy and error in diagnostic radiology. WEMJ. 2012;111(2):4.

Hostetter R, Nakazawa N, Tompkins K, Hill B. Precision in central venous catheter tip placement: a review of the literature. J Assoc Vasc Access. 2010;15(3):112-25.

Ibrahim G. Central venous placement: where is the tip? Am J Crit Care. 2012;21(5):370-1.

Johnston AJ, Bishop SM, Martin L, See TC, Streater CT. Defining peripherally inserted central catheter tip position and an evaluation of insertions in one unit. Anaesthesia. 2013;68(5):484-91. 
Kung SC, Aravind B, Morse S, Jacobs LE, Raja R. Tunneled catheter-associated atrial thrombi: successful treatment with chronic anticoagulation. Hemodial Int. 2001;5(1):32-6.

Liu Y-J, Dong L, Lou X-P, Miao J-H, Li X-X, Li X-J, Li J, Liu Q-Q, Chang Z-W. Evaluating ECG-aided tip localization of peripherally inserted central catheter in patients with cancer. Int J Clin Exp Med. 2015;8(8):14127-9.

Mandolfo S, Piazza W, Galli F. Catheter tip position and haemodialysis patient; a difficult symbiosis. J Vasc Access. 2002;3(2):64-73.

Marano L, Izzo G, Esposito G, Petrillo M, Cosenza A, Marano M, et al. Peripherally inserted central catheter tip position: a novel empirical-ultrasonographical index in a modern surgical oncology department. Ann Surg Oncol. 2014;21(2):656-61.

Marey EJ. Des variations électriques des muscles et du coeur en particulier, étudiées au moyen de l'électromètre de M. Lippmann. CR Acad Sci. 1876;82:975-7.

Massmann A, Jagoda P, Kranzhoefer N, Buecker A. Local low-dose thrombolysis for safe and effective treatment of venous port-catheter thrombosis. Ann Surg Oncol. 2015;22(5):1593-7.

Matteucci C. Sur un phenomene physiologique produit par les muscles en contraction. Ann Chim Phys. 1842;6:339-41.

Mehall J, Saltzman D, Jackson R, Smith S. Fibrin sheath enhances central venous catheter infection. Crit Care Med. 2002;30(4):908-12.

Miller-Keane. Encyclopedia and dictionary of medicine, nursing, and allied health. 7th ed. 2003. http://medical-dictionary.thefreedictionary.com/parallax+effect. Accessed 24 May 2017.

Moureau NL. Vessel health and preservation: vascular access assessment, selection, insertion, management, evaluation and clinical education. Doctor of Philosophy by Publication (PhD) Thesis and Exegesis (PhD Doctorate), Griffith University. 2017. https:// www120.secure.griffith.edu.au/rch/items/e6aea329fae8-4c41-aa3f-6b4f80298977/1/. https://www120. secure.griffith.edu.au/rch/items/e6aea329-fae8-4c41aa3f-6b4f80298977/1/ (gu1507011216006).

Moureau N, Chopra V. Indications for peripheral, midline, and central catheters: summary of the michigan appropriateness guide for intravenous catheters recommendations. J Assoc Vasc Access. 2016;21: $140-8$.

Moureau N, Dennis G, Ames E, Severe R. Electrocardiogram (EKG) guided peripherally inserted central placement and tip position: results of a trial to replace radiological confirmation. J Assoc Vasc Access. 2010;15(1):9-15. https://doi.org/10.2309/ java.15-1-3

National Association of Vascular Access Networks. NAVAN position statement on terminal tip placement. J Vasc Access Devices. 1998;3:8-10.
NKF-K/DOQI. Clinical practice guidelines for vascular access. Am J Kidney Dis. 2001;37(1):S137-S18. https://doi.org/10.1016/S0272-6386(01)70007-8.

Oliver G, Jones M. ECG or X-ray as the 'gold standard' for establishing PICC-tip location? Br J Nurs. 2014;23(Suppl 19):S10-6. https://doi.org/10.12968/ bjon.2014.23.Sup19.S10.

Petersen J, Delaney J, Brakstad M, Rowbotham R, Bagley C. Silicone venous access devices positioned with their tips high in the superior vena Caba are more likely to malfunction. Am J Surg. 1999;178(1):38-41.

Pittiruti M. Sweet Spot' vs. Carina: two criteria for verifying tip location by chest X-ray. J Assoc Vasc Access. 2015;20(4):240-1.

Pittiruti M, Scoppettuolo G, LaGreca A, Emoli A, Brutti A, Migliorini I, et al. The EKG method for positioning the tip of PICCs: results from two preliminary studies. J Assoc Vasc Access. 2008;13(4):179-86. https://doi. org/10.2309/ava.13-4-4.

Pittiruti M, Hamilton H, Biffi R, MacFie J, Pertkiewicz M. ESPEN guidelines on parenteral nutrition: central venous catheters (access, care, diagnosis and therapy of complications). Clin Nutr. 2009;28(4):365-77. https://doi.org/10.1016/j.clnu.2009.03.015.

Pittiruti M, La Greca A, Scoppettuolo G. The electrocardiographic method for positioning the tip of central venous catheters. J Vasc Access. 2011;12(4):280-91. https://doi.org/10.5301/JVA.2011.8381.

Pittiruti M, Bertollo D, Briglia E, Buononato M, Capozzoli G, De Simone L, et al. The intracavitary ECG method for positioning the tip of central venous catheters: results of an Italian Multicenter study. J Vasc Access. 2012;13(3):357-65.

Pittiruti M, LaGreca A, Emoli A, Calabrese M, Biasucci DG, Scoppettuolo G. Tip location of central venous access in patients with atrial fibrillation and pacemakers: an algorithm minimizing X-RAY exposure. J Assoc Vasc Access. 2014;19(4):210-1.

Raad I, Bodey G. Infectious complications of indwelling vascular catheters. Clin Infect Dis. 1992;15(2):197-208.

Raman D, Sharma M, Moghekar A, Wang X, Hatipoğlu U. Utilization of thoracic ultrasound for confirmation of central venous catheter placement and exclusion of pneumothorax: a novel technique in real-time application. J Intensive Care Med. 2017;0885066617705839.

RCN. Standards for infusion therapy. 4th ed. Royal College of Nursing; 2016. p. 1-94.

Roldan C, Paniagua L. Central venous catheter intravascular malpositioning: causes, prevention, diagnosis, and correction. West J Emerg Med. 2015;16(5):658-64.

Rossetti F, Pittiruti M, Lamperti M, Graziano U, Celentano D, Capozzoli G. The intracavitary ECG method for positioning the tip of central venous access devices in pediatric patients: results of an Italian multicenter study. J Vasc Access. 2015;16(2):137-43. https://doi. org/10.5301/jva.5000281.

Ryder M. The role of biofilm in vascular catheter-related infections. N Dev Vasc Dis. 2001;2:15-25. 
Ryu HG, Bahk JH, Kim JT, Lee JH. Bedside prediction of central venous catheter insertion depth. Br J Anaesth. 2007;98(2):225-7.

Sansivero GE. What's new in vascular access devices and technology? Br J Nurs. 2012;21(Suppl 1):S16-20.

Schummer W, Schummer C, Schelenz C, Brandes H, Stock U, Muller T, et al. Central venous catheters-the inability of intra-atrial ECG to prove adequate positioning. Br J Anaesth. 2004;93(2):193-8.

Schuster M, Nave H, Piepenbrock S, Pabst R, Panning $\mathrm{B}$. The Carina as a landmark in central venous catheter placement. Br J Anaesth. 2000;85(2):192-4.

Scott W. Central venous catheters. An overview of Food and Drug Administration activities. Surg Oncol Clin N Am. 1995;4(3):377-93.

Sette P, Azzini AM, Dorizzi RM, Castellano G. Serendipitous ECG guided PICC insertion using the guidewire as intra-cardiac electrode. $\mathrm{J}$ Vasc Access. 2010;11(1):72.

Smith B, Neuharth RM, Hendrix MA, McDonnall D, Michaels AD. Intravenous electrocardiographic guidance for placement of peripherally inserted central catheters. J Electrocardiol. 2010;43(3):274-8. https:// doi.org/10.1016/j.jelectrocard.2010.02.003.

Tal M, Friedman T, MojiBian H. The Function tip: a different look at a contentious topic. Endovasc Today. 2013;73-5.

Tortora G, Derrickson B. Principles of anatomy and physiology. 11th ed. Wiley; 2006.

Verhey P, Gosselin M, Primack S, Blackburn P, Kraemer A. The right mediastinal border and central venous anatomy on frontal chest radiograph-direct CT correlation. J Assoc Vasc Access. 2008;13(1):32-5.

Vesely T. Central venous catheter tip position: a continuing controversy. J Vasc Interv Radiol. 2003;14(5):527-34.

Walker G, Alexandrou E, Rickard CM, Chan RJ, Webster J. Effectiveness of electrocardiographic guidance in CVAD tip placement. Br J Nurs. 2015;24(Suppl 14):S4-S12.

Waller AD. A demonstration on man of electromotive changes accompanying the heart's beat. J Physiol. 1887;8(5):229-34.

Wallis M, McGrail M, Webster J, Marsh N, Gowardman J, Playford E, Rickard C. Risk factors for peripheral intravenous catheter failure: a multivariate analysis of data from a randomized controlled trial. Infection Control Hospital Epidemiology. 2014;35(1):63-8. https://doi.org/10.1086/674398.

Weekes AJ, Keller SM, Efune B, Runyon M. Prospective comparison of ultrasound and CXR for confirmation of central venous catheter placement. Emerg Med J. 2016;33(3):176-80.

Wolters, Kluwer, Lippincott. ECG interpretation: an incredibly easy pocket guide. Published Book; 2009.

Wuerz L, Cooke J, Hentel K, Ince-Barnes J, Dawson R. Successful implementation of electrocardiogramplaced peripherally inserted central catheters at a major academic medical teaching organization. $\mathrm{J}$ Assoc Vasc Access. 2016;21(4):223-9.

Open Access This chapter is licensed under the terms of the Creative Commons Attribution 4.0 International License (http://creativecommons.org/licenses/by/4.0/), which permits use, sharing, adaptation, distribution and reproduction in any medium or format, as long as you give appropriate credit to the original author(s) and the source, provide a link to the Creative Commons license and indicate if changes were made.

The images or other third party material in this chapter are included in the chapter's Creative Commons license, unless indicated otherwise in a credit line to the material. If material is not included in the chapter's Creative Commons license and your intended use is not permitted by statutory regulation or exceeds the permitted use, you will need to obtain permission directly from the copyright holder. 